\title{
Spin-dependent coupling between quantum dots and topological quantum wires
}

\author{
Silas Hoffman, Denis Chevallier, Daniel Loss, and Jelena Klinovaja \\ Department of Physics, University of Basel, Klingelbergstrasse 82, CH-4056 Basel, Switzerland
}

(Received 8 May 2017; published 31 July 2017)

\begin{abstract}
Considering Rashba quantum wires with a proximity-induced superconducting gap as physical realizations of Majorana bound states and quantum dots, we calculate the overlap of the Majorana wave functions with the local wave functions on the dot. We determine the spin-dependent tunneling amplitudes between these two localized states and show that we can tune into a fully spin polarized tunneling regime by changing the distance between dot and Majorana bound state. Upon directly applying this to the tunneling model Hamiltonian, we calculate the effective magnetic field on the quantum dot flanked by two Majorana bound states. The direction of the induced magnetic field on the dot depends on the occupation of the nonlocal fermion formed from the two Majorana end states which can be used as a readout for such a Majorana qubit.
\end{abstract}

DOI: 10.1103/PhysRevB.96.045440

\section{INTRODUCTION}

Majorana bound states (MBSs) [1-8] are promising candidates for topological quantum computation. Being spinless and chargeless particles, they are robust to disorder [9-12]. However, these properties that make them a desirable element for information storage make readout problematic. Nonetheless, there have been several schemes for storage, manipulation, and readout of topological quantum computers using MBSs modeled as a Kitaev chain [13], which is largely phenomenological [14-22]. A theoretical analysis of physically realized MBSs for quantum information storage has yet to be rigorously studied; the details of which, as we show in this paper, are critical for quantum operations.

Although there are several systems in which MBSs have been proposed, perhaps the most readily accessible are quantum wires [23,24] because: (1) there is potentially a large spin-orbit interaction (SOI), (2) advances in material science allow superconductivity to be easily induced by proximity, and (3) electrical gating allows the wire to be easily tuned in and out of the topological regime (see for instance Ref. [25]). When two ends of two quantum wires are brought close to each other, the two MBSs at the ends form a nonlocal fermionic state which can be occupied or unoccupied. If a quantum dot [26,27], which can be electrically defined in experiments within the same quantum wire, is brought into proximity of these Majorana end states, the charge or spin coupling can be used to readout the parity of the quantum wire junction; such a scheme was proposed in Ref. [19] wherein MBSs, described by a Kitaev chain, were coupled to a spinful single-level quantum dot and later adapted to a spinless single-level dot in Refs. [21,22].

In this paper we study MBSs formed in a quantum wire with proximity-induced superconductivity near a quantum dot [28-37], which is also formed inside a quantum wire, all of which are subject to an applied magnetic field perpendicular to the SOI. First calculating the wave functions of the quantum dot and MBSs, which can be done analytically in the strong spin-orbit regime, we are able to find the spin dependence of the quantum dot-MBS coupling which has been largely neglected $[21,22,28,29]$. We find that by changing the relative position between dot and MBSs, one can tune between spin-independent and fully polarized tunneling for typical parameters. In the presence of two MBSs, the spin-dependent tunneling induces an effective magnetic field on the dot which changes direction when the occupation of the nonlocal fermion, formed from the two MBSs, changes parity. Thus, this setup allows the readout of MBS qubits via reading out the spin of the electron on the quantum dot [19].

We organize the paper as follows: in Sec. II we describe the quantum wire that hosts two MBSs and a quantum dot. In Sec. III we calculate the overlap of the MBS and quantum dot wave functions, and thus the spin-dependent tunneling, for which we obtain simple analytic expressions in a suitable limit. Using these results, in Sec. IV we consider two MBSs coupled to the dot and calculate the effective magnetic field when the complex fermion state formed from the MBSs is occupied or unoccupied. In Sec. V we numerically calculate the spin-dependent coupling and the effective magnetic field on the dot using a tight-binding model. We conclude in the final section with a summary of our results and their implications on proposed quantum dot-MBS computational schemes.

\section{MODEL}

We consider a quantum wire in proximity to a conventional superconductor, so superconducting pairing is induced, and a magnetic field along the longitudinal axis which is perpendicular to the spin-orbit direction (see Fig. 1). There is full spatial control of the chemical potential over the wire so that the right section and left section are tuned into the topological and nontopological regimes, respectively, with appropriate gating; at the intersection resides a MBS. Within the nontopological section, appropriate gates define a quantum dot which supports a localized wave function. For a sufficiently large barrier between the MBS and quantum dot, which we assume in the following, the MBS wave function can be solved independently from the quantum dot wave functions within the same quantum wire. For the remainder of the paper we assume the temperature to be above the Kondo temperature.

\section{A. Majorana bound state}

To find the MBS wave function, we consider the two sections in the quantum wire, topological $(v=t)$ and nontopological $(v=n)$, to be kept at different chemical potentials, $\mu_{n}$ 


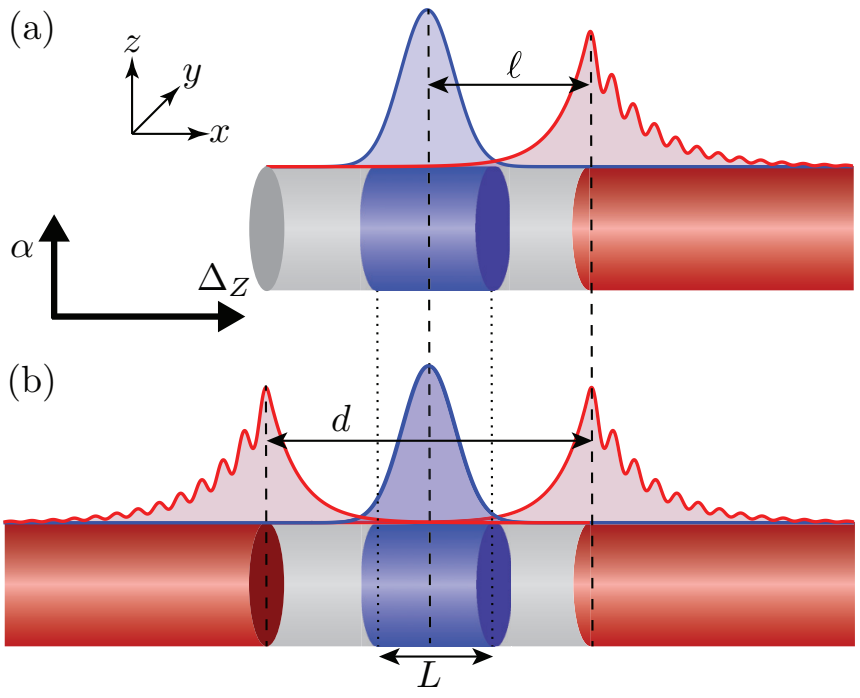

FIG. 1. A quantum wire with applied magnetic field along the longitudinal $(x)$ axis and spin-orbit vector along the $z$ axis in which the boundary between a topological section (red) and nontopological section (gray) supports a MBS. In the upper panel, a quantum dot (blue) of size $L$ is defined, within the nontopological section, at a distance $\ell$ from the topological section. The second setup (lower panel) is identical to the first with an additional topological section which ends a distance $d-\ell$ from the quantum dot center. The red and blue curves are schematically the probability amplitudes of the MBS and quantum dot wave functions, respectively.

and $\mu_{t}$, and whose interface is at $x=\ell$. The Hamiltonian of this system is

$$
H=\int d x \Psi^{\dagger}(x)\left(\mathcal{H}_{0}+\mathcal{H}_{\mathrm{SO}}+\mathcal{H}_{s}+\mathcal{H}_{Z}\right) \Psi(x),
$$

where

$$
\begin{aligned}
& \mathcal{H}_{0}=-\eta_{3}\left[\hbar^{2} \partial_{x}^{2} / 2 m+\mu(x)\right], \quad \mathcal{H}_{\mathrm{SO}}=-i \alpha \sigma_{3} \partial_{x}, \\
& \mathcal{H}_{Z}=\Delta_{Z} \sigma_{1} \eta_{3}, \quad \mathcal{H}_{s}=\Delta_{s} \sigma_{2} \eta_{2}
\end{aligned}
$$

are the kinetic, SOI interaction, Zeeman splitting, and superconducting pairing, terms, respectively. Here $\Psi(x)=$ $\left[\psi_{\uparrow}(x), \psi_{\downarrow}(x) \psi_{\uparrow}^{\dagger}(x), \psi_{\downarrow}^{\dagger}(x)\right]^{T}$ is the Nambu spinor in the quantum wire, $\mu(x)=\mu_{t} \Theta(x-\ell)+\mu_{n} \Theta(\ell-x)$, $\alpha$ is the SOI constant, $\Delta_{Z}$ is the Zeeman splitting due to the applied magnetic field, $\Delta_{S}$ is the proximity induced superconducting gap, and $\psi_{\sigma}(x)\left[\psi_{\sigma}^{\dagger}(x)\right]$ annihilates (creates) an electron of spin $\sigma=\uparrow, \downarrow$ quantized along the $z$ axis at position $x$. The Pauli matrices $\sigma_{i}$ and $\eta_{i}$ act in spin and particle-hole space, respectively. The condition $\Delta_{Z}^{2}>\mu_{t}^{2}+\Delta_{s}^{2}$ is necessary to be in the topological phase [23,24,38,39].

In the following, we consider the SOI energy to be large [40] compared to the magnetic field $\left(E_{\mathrm{SO}}=m \alpha^{2} / \hbar^{2} \gg \Delta_{Z}\right)$ and the superconducting gap $\left(E_{\mathrm{SO}} \gg \Delta_{S}\right)$. We tune the right section of the wire into the topological regime by fixing the chemical potential to zero, $\mu_{t}=0$, and applying a large enough magnetic field such that the Zeeman splitting is larger than the superconducting gap, i.e., $\Delta_{Z}>\Delta_{s}$. We consider two ways in which the left section can be driven into the nontopological regime: (1) chemical potential is small compared to the SOI energy but large enough so that $\Delta_{Z}^{2}<\Delta_{s}^{2}+\mu_{n}^{2}$, or
(2) a fully depleted wire, $-\mu_{n} \gg E_{\text {SO }} \gg \Delta_{Z}, \Delta_{s}$, which is insulating in the normal phase. Although the second regime presents a more physical experimental realization [26], we are unable to analytically progress beyond the zero bulk solutions to the Hamiltonian, i.e., we cannot satisfy differentiability of the MBS wave functions at the boundary (Appendix A). Therefore, we consider the former case which yields simple analytic results that are instructive in guiding the numerical methods used to solve the system in the latter regime (see below).

When the chemical potential is much smaller than the SOI energy, it is standard to go to the rotating frame of reference [41], dropping fast oscillating terms, to obtain a linearized Hamiltonian [39]. Rotating back to the laboratory frame, the zero energy eigenfunctions are given by (see Appendix A)

$$
\begin{aligned}
& \Phi_{1}^{v}=\left(\begin{array}{c}
-i \operatorname{sgn}\left(\Delta_{s}-\sqrt{\Delta_{Z}^{2}-\mu_{v}^{2}}\right) e^{i \varphi_{\nu} / 2} \\
e^{-i \varphi_{\nu} / 2} \\
i \operatorname{sgn}\left(\Delta_{s}-\sqrt{\Delta_{Z}^{2}-\mu_{v}^{2}}\right) e^{-i \varphi_{v} / 2} \\
e^{i \varphi_{v} / 2}
\end{array}\right) e^{-\kappa_{1}^{v}(x-\ell)}, \\
& \Phi_{2}^{v}=\left(\begin{array}{c}
e^{-i \varphi_{\nu} / 2} \\
-i e^{i \varphi_{\nu} / 2} \\
e^{i \varphi_{\nu} / 2} \\
i e^{-i \varphi_{\nu} / 2}
\end{array}\right) e^{-\kappa_{2}^{\nu}(x-\ell)}, \\
& \Phi_{3}^{\nu}=\left(\begin{array}{c}
i e^{2 i k_{\mathrm{SO}}(x-\ell)} \\
e^{-2 i k_{\mathrm{SO}}(x-\ell)} \\
-i e^{-2 i k_{\mathrm{SO}}(x-\ell)} \\
e^{2 i k_{\mathrm{SO}}(x-\ell)}
\end{array}\right) e^{-\kappa^{\nu}(x-\ell)}, \\
& \Phi_{4}^{v}=\left(\begin{array}{c}
e^{2 i k_{\mathrm{SO}}(x-\ell)} \\
i e^{-2 i k_{\mathrm{SO}}(x-\ell)} \\
e^{-2 i k_{\mathrm{SO}}(x-\ell)} \\
-i e^{2 i k_{\mathrm{SO}}(x-\ell)}
\end{array}\right) e^{-\kappa^{\nu}(x-\ell)},
\end{aligned}
$$

where $\quad \kappa_{1}^{v}= \pm\left|\Delta_{s}-\sqrt{\Delta_{Z}^{2}-\mu_{v}^{2}}\right| / \alpha, \quad \kappa_{2}^{v}= \pm\left(\Delta_{s}+\right.$ $\left.\sqrt{\Delta_{Z}^{2}-\mu_{\nu}^{2}}\right) / \alpha, \quad \kappa^{v}= \pm \Delta_{s} / \alpha, \quad$ and $\sin \varphi_{\nu}=\mu_{\nu} / \Delta_{Z}$ for which we require $\Delta_{Z} \geqslant \mu_{\nu}$. The \pm in the real part of the exponentials refers to $v=t, n$, respectively, and $k_{\text {SO }}=m \alpha / \hbar^{2}$ is the SOI wave vector. Here we have neglected terms $\mu_{n} / \alpha \ll k_{\text {SO }}$ in the wave functions $\Phi_{3}^{n}$ and $\Phi_{4}^{n}$ which renormalize the oscillations due to a shift in the Fermi points.

The wave functions in Eq. (3) are zero-energy eigenvectors of the Hamiltonian but do not individually satisfy the boundary conditions. The MBS wave function satisfying continuity and differentiability at the boundary is $\Phi_{M}=\Theta(x-\ell) \Phi^{t}+$ $\Theta(\ell-x) \Phi^{n}$, where

$$
\begin{aligned}
\Phi^{t} & =\mathcal{N}\left(\Phi_{1}^{t}-\frac{\kappa_{1}^{t}+\kappa^{t}}{2 \kappa^{t}} \Phi_{3}^{t}+\frac{k_{\mathrm{SO}}}{\kappa^{t}} \Phi_{4}^{t}\right), \\
\Phi^{n} & =\mathcal{N}\left(\frac{\kappa^{t}-\kappa_{1}^{t}}{2 \kappa^{t}} \Phi_{3}^{n}+\frac{k_{\mathrm{SO}}}{\kappa^{t}} \Phi_{4}^{n}\right)
\end{aligned}
$$

and where $\mathcal{N}$ is an overall normalization factor. See Appendix A for a full derivation of Eq. (4). The probability amplitude of the MBS, $\left|\Phi_{M}\right|^{2}$, on the topological section oscillates with half 
the spin-orbit wavelength, $\lambda_{\mathrm{SO}} / 2=\pi / k_{\mathrm{SO}}$, and has two decay lengths given by the superconducting gap $\Delta_{s}$ and the induced gap $\left|\Delta_{Z}-\Delta_{S}\right|$. On the nontopological section, although the components of the MBS wave function oscillate with the same $\lambda_{\text {so }} / 2$ periodicity, the probability amplitude is a monotonically decreasing exponential with decay length $\alpha / \Delta_{s}$ [see Fig. 1 (red part)]. The shape of MBS wave functions could be mapped experimentally using the STM techniques $[7,8,42,43]$.

\section{B. Quantum dot}

There are two characteristic regimes in which one can create the quantum dot: (1) when the dot size is smaller than the spin-orbit length $l_{\mathrm{SO}}=1 / k_{\mathrm{SO}}$, and (2) when it is larger. In the first case, the SOI term can be neglected while in the second case the spin components of the wave function oscillate on the wave vector $k_{\text {SO }}$ [44]. Experimentally, the spatial profile of the superconductivity and gates between the quantum dot and MBS wave functions could be complicated. However, we expect this to contribute only a spin independent factor to the tunneling, which can be absorbed as a phenomenological parameter. In order to simplify the calculation, we consider a fully depleted section of the wire so that we can ignore the superconducting correlations on the dot. This allows us to analytically calculate the dot wave function and thus the spin-dependent tunneling which is the focus of the paper.

Small dot-In the first case, the dot is described by

$$
H_{D}^{s}=\int d x \Psi^{\dagger}(x)\left[\mathcal{H}_{0}+\mathcal{H}_{Z}+\mathcal{V}(x)\right] \Psi(x),
$$

where $\mathcal{V}(x)$ is a confining potential defining the dot. For a parabolic confinement, $\mathcal{V}(x)=m \omega_{0}^{2} x^{2} / 2-\mu_{d}$, where $\mu_{d}$ is a dot plunger potential, the lowest energy eigenvectors of $H_{D}^{s}$ are $X^{i}(x)=\left(1 / 4 \pi L^{2}\right)^{1 / 4} \exp \left(-x^{2} / 2 L^{2}\right) \chi^{i}$, where $L=\sqrt{\hbar / m \omega_{0}}$ and

$$
\begin{gathered}
\chi^{1}=\left(\begin{array}{l}
1 \\
1 \\
0 \\
0
\end{array}\right), \quad \chi^{2}=\left(\begin{array}{c}
-1 \\
1 \\
0 \\
0
\end{array}\right), \\
\chi^{3}=\left(\begin{array}{l}
0 \\
0 \\
1 \\
1
\end{array}\right), \quad \chi^{4}=\left(\begin{array}{c}
0 \\
0 \\
-1 \\
1
\end{array}\right),
\end{gathered}
$$

with eigenenergies

$$
\begin{aligned}
\epsilon_{\uparrow} & =\epsilon_{0}-\Delta_{Z}, \quad \epsilon_{\downarrow}=\epsilon_{0}+\Delta_{Z}, \\
-\epsilon_{\uparrow} & =-\epsilon_{0}+\Delta_{Z}, \quad-\epsilon_{\downarrow}=-\epsilon_{0}-\Delta_{Z},
\end{aligned}
$$

respectively, and $\epsilon_{0}=\hbar \omega_{0} / 2-\mu_{d}$.

Large dot-In the second case, the Hamiltonian is

$$
H_{D}^{l}=\int d x \Psi^{\dagger}(x)\left[\mathcal{H}_{0}+\mathcal{H}_{\mathrm{SO}}+\mathcal{H}_{Z}+\mathcal{V}(x)\right] \Psi(x),
$$

where $\mathcal{H}_{\mathrm{SO}}$ is the SOI as given in Sec. II A and $\mathcal{V}(x)$ is a parabolic confinement as in the case of the small dot. Treating the magnetic field perturbatively as compared to the other energies on the dot, one may show that the Hamiltonian reduces to Eq. (5) in the rotating frame of reference with an exponentially renormalized magnetic field according to ratio of the dot size and SOI length $\bar{\Delta}_{Z}=\Delta_{Z} e^{-k_{\mathrm{SO}} L}$ [44]. The eigenvectors are $Y^{i}(x)=\left(1 / 4 \pi L^{2}\right)^{1 / 4} \exp \left(-x^{2} / 2 L^{2}\right) \zeta^{i}$, where

$$
\begin{aligned}
& \zeta^{1}=\left(\begin{array}{c}
e^{i k_{\mathrm{SO}} x} \\
e^{-i k_{\mathrm{SO}} x} \\
0 \\
0
\end{array}\right), \quad \zeta^{2}=\left(\begin{array}{c}
-e^{i k_{\mathrm{SO}} x} \\
e^{-i k_{\mathrm{SO}} x} \\
0 \\
0
\end{array}\right), \\
& \zeta^{3}=\left(\begin{array}{c}
0 \\
0 \\
e^{-i k_{\mathrm{SO}} x} \\
e^{i k_{\mathrm{SO}} x}
\end{array}\right), \quad \zeta^{4}=\left(\begin{array}{c}
0 \\
0 \\
-e^{-i k_{\mathrm{SO}} x} \\
e^{i k_{\mathrm{SO}} x}
\end{array}\right),
\end{aligned}
$$

with eigenenergies, respectively,

$$
\epsilon_{0}-\bar{\Delta}_{Z}, \quad \epsilon_{0}+\bar{\Delta}_{Z}, \quad-\epsilon_{0}-\bar{\Delta}_{Z}, \quad-\epsilon_{0}+\bar{\Delta}_{Z} .
$$

\section{SPIN-DEPENDENT TUNNELING}

It is now straightforward to evaluate the tunneling amplitudes between the MBS and quantum dot states which are proportional to the overlap of the two corresponding wave functions,

$$
\begin{aligned}
& t_{\uparrow}=\bar{t}_{0} \int d x\left(X^{1}\right)^{\dagger} \Phi_{M}=\bar{t}_{0}\left(\int d x\left(X^{3}\right)^{\dagger} \Phi_{M}\right)^{*}, \\
& t_{\downarrow}=\bar{t}_{0} \int d x\left(X^{2}\right)^{\dagger} \Phi_{M}=\bar{t}_{0}\left(\int d x\left(X^{4}\right)^{\dagger} \Phi_{M}\right)^{*},
\end{aligned}
$$

where $\bar{t}_{0}$ is a phenomenological constant that is defined according to the potential profile separating the dot and MBS. The tunneling Hamiltonian between the dot and MBS is $[16,19]$

$$
H_{T}=\sum_{\sigma} t_{\sigma} d_{\sigma}^{\dagger} \gamma+\text { H.c. }
$$

where $\gamma$ is the MBS operator and $d_{\sigma}^{\dagger}$ creates an electron with spin $\sigma$, quantized along the axis of the magnetic field ( $x$ axis).

In the limit that $k_{\mathrm{SO}} L \ll 1$, which also implies that $L$ is much smaller than the MBS decay lengths in the problem, the tunneling amplitudes are

$$
\begin{aligned}
& \frac{t_{\uparrow}}{t_{0}} \approx(1+i) \cos \left(2 k_{\mathrm{SO}} \ell+\pi / 4\right) e^{-\kappa^{t} \ell}, \\
& \frac{t_{\downarrow}}{t_{0}} \approx-(1-i) \cos \left(2 k_{\mathrm{SO}} \ell-\pi / 4\right) e^{-\kappa^{t} \ell},
\end{aligned}
$$

where the approximation neglects terms of order $1, \kappa_{1}^{t} / \kappa^{t} \ll$ $k_{\mathrm{SO}} / \kappa^{t}$, and $t_{0}=\left(\pi L^{2} / 4\right)^{1 / 4} k_{\mathrm{SO}} \mathcal{N} \bar{t}_{0} / \kappa^{t}$ is the renormalized phenomenological constant which fixes the maximum tunneling. The functions in Eq. (13) are plotted in Fig. 2 as a function of distance between the dot and topological section $\ell$; distances are measured in units of spin-orbit length $l_{\mathrm{SO}}=k_{\mathrm{SO}}^{-1}$, though only the dimensionless product of lengths and wave vectors is relevant. Notice that $\operatorname{Re}\left[t_{\uparrow}\right]=\operatorname{Im}\left[t_{\uparrow}\right]$ and $\operatorname{Re}\left[t_{\downarrow}\right]=-\operatorname{Im}\left[t_{\uparrow}\right]$, both of which decay exponentially with $\kappa^{t} \ell$ and oscillate with the period $\lambda_{\mathrm{so}} / 2$, which can be attributed to the relative factor of $e^{2 i k_{\mathrm{SO}} x}$ between the dot and MBS wave functions. Furthermore, because there is a difference in phase of $\pi / 2$ between $t_{\uparrow}$ and $t_{\downarrow}$, by changing the distance between the dot and MBS, the tunneling can go from full polarization of one 


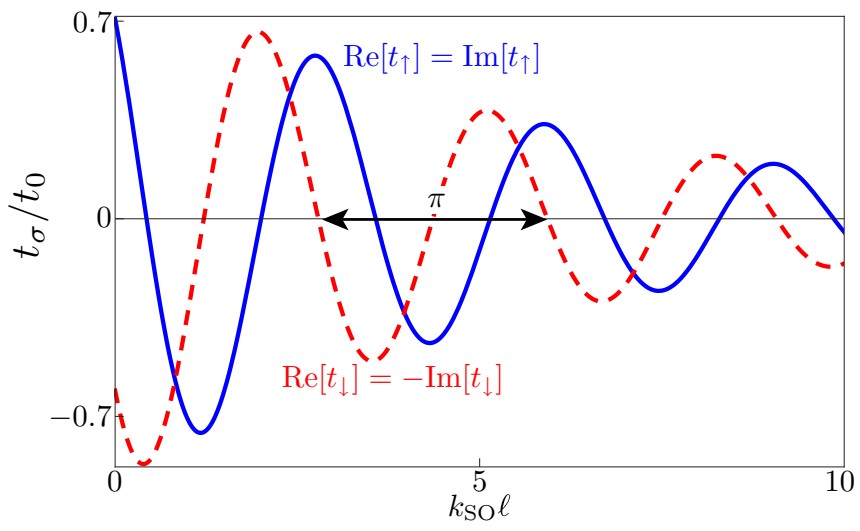

FIG. 2. The real and imaginary components of spin-dependent tunneling amplitudes $t_{\uparrow}$ (blue solid) and $t_{\downarrow}$ (red dashed) for a small quantum dot, $k_{\mathrm{SO}} L=0.1$, and $\kappa^{t} / k_{\mathrm{SO}}=10 \sqrt{3}$ as a function of distance $\ell$ between the dot and the topological section. Both $t_{\uparrow}$ and $t_{\downarrow}$ oscillate with periodicity of $\lambda_{\mathrm{SO}} / 2$ but with a relative phase difference of $\pi / 2$.

spin, either $t_{\uparrow}=0$ or $t_{\downarrow}=0$, to equal magnitude spin tunneling $\left|t_{\uparrow}\right|=\left|t_{\downarrow}\right|$.

When the dot size is comparable to the spin orbit length $k_{\mathrm{SO}} L \gtrsim 1$, there is no simple analytical formula for the tunneling coefficients. Upon comparing the dot and MBS wave functions, there is a relative factor of $e^{i k_{\mathrm{SO}} x}$ and we therefore expect the tunneling amplitudes to oscillate with the period $\lambda_{\text {so }}$, which is twice that of the small dot. Indeed, in Fig. 3 we see an exponential decrease as a function of $\ell$ and oscillatory behavior with period $\lambda_{\text {SO }}$ with the spin up and down components differing in phase by $\pi / 2$. Because the wave function of the dot is more extended, the overlap of dot and MBS wave functions is reduced, as compared to the small dot case, so that the maximum magnitude of $t_{\sigma} / t_{0}$ is small; the magnitude of $t_{\sigma}$ can be increased by increasing $\bar{t}_{0}$

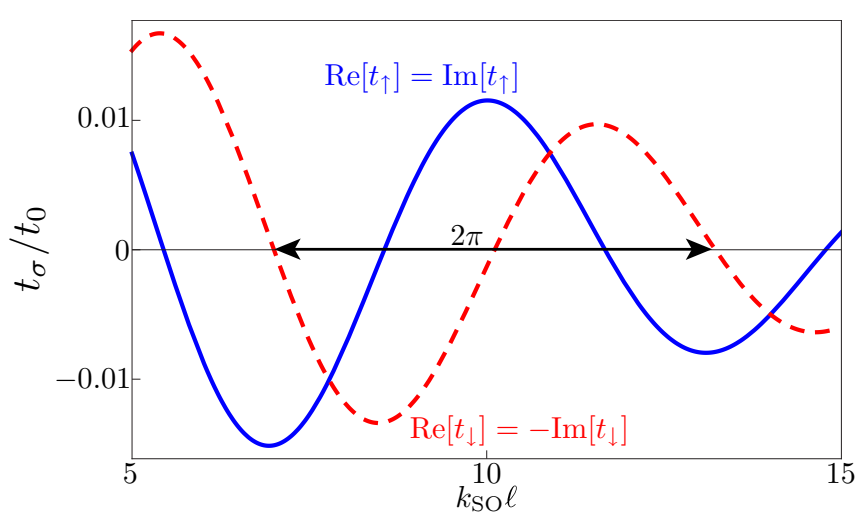

FIG. 3. The real and imaginary components of spin-dependent tunneling amplitudes $t_{\uparrow}$ (blue solid) and $t_{\downarrow}$ (red dashed) for a large dot, $k_{\mathrm{SO}} L=10$ and $\kappa^{t} / k_{\mathrm{SO}}=10 \sqrt{3}$, as a function of distance $\ell$ between the dot and the topological section. Both $t_{\sigma}$ oscillate with the period $\lambda_{\text {So }}$ and the magnitude of oscillation is smaller as compared with the case of a small dot. Due to the finite size of the dot, we start with a separation $\ell=L / 2$ between the center of the dot and the end of the topological section. which roughly corresponds to decreasing the barrier between the topological end and the quantum dot in an experiment.

\section{EFFECTIVE MAGNETIC FIELD}

In this section we extend our setup by considering two ends of identical topological superconductor sections, separated by a distance $d$, flanking opposite sides of a quantum dot [see Fig. 1(b)], at a distance $\ell$ from the right MBS. Because the MBS wave functions are symmetric at the ends of either topological superconductor section, the overlap of the right tunneling amplitudes are given by Eq. (13), $t_{\sigma r}=t_{\sigma}$, while the left tunneling amplitudes are analogously given by

$$
\begin{aligned}
& \frac{t_{\uparrow l}}{t_{0}} \approx(1-i) \cos \left[2 k_{\mathrm{SO}}(d-\ell)+\pi / 4\right] e^{-(d-\ell) \kappa^{t}}, \\
& \frac{t_{\downarrow l}}{t_{0}} \approx-(1+i) \cos \left[2 k_{\mathrm{SO}}(d-\ell)-\pi / 4\right] e^{-(d-\ell) \kappa^{t}} .
\end{aligned}
$$

Here we neglect any direct overlap between MBSs in the wire $[45,46]$ or via the bulk superconductor [47]. The corresponding tunneling Hamiltonian is written as [19]

$$
H_{T}=\sum_{\sigma, \lambda} t_{\sigma \lambda} d_{\sigma}^{\dagger} \gamma_{\lambda}+\text { H.c. },
$$

where $\lambda=l, r$ specifies the left and right MBS, respectively. Following Ref. [19], we find that a Schrieffer-Wolff transformation yields [48], to second order in tunneling,

$$
\mathcal{H}_{T}=\sum_{i=0, x, y, z}\left(B_{i}^{-} f f^{\dagger}+B_{i}^{+} f^{\dagger} f\right) S_{i},
$$

where $f=\gamma_{r}+i \gamma_{l}\left(f^{\dagger}=\gamma_{r}-i \gamma_{l}\right)$ is the nonlocal fermionic annihilation (creation) operator formed from the MBSs. We remind the reader that the axis of quantization here is the dot axis, along the applied magnetic field ( $x$ axis), which is related to the wire axis of quantization, along the spin orbit direction ( $z$ axis), by a $\pi / 2$ rotation around the $y$ axis; hence the spin on the dot, in the frame defined in Fig. 1, is $S_{i}=\sum_{\sigma \sigma^{\prime}} d_{\sigma}^{\dagger}\left(R_{y}^{\dagger} \sigma^{i} R_{y}\right)_{\sigma \sigma^{\prime}} d_{\sigma^{\prime}}$ with $\sigma^{0}=\mathbb{1}_{2 \times 2}$ and $R_{y}=$ $\left(\sigma^{0}-i \sigma^{y}\right) / \sqrt{2}$. According to Eq. (16), a different overall shift in energy and effective magnetic field is exerted on the quantum dot when the fermionic state is occupied $\left(B_{i}^{+}\right)$or unoccupied $\left(B_{i}^{-}\right)$, where (see Appendix B)

$$
\begin{aligned}
& B_{0}^{ \pm}=\frac{\left|t_{\uparrow \pm}\right|^{2}}{\epsilon_{\uparrow} \pm 2 \delta}+\frac{\left|t_{\downarrow \pm}\right|^{2}}{\epsilon_{\downarrow} \pm 2 \delta}, \\
& B_{x}^{ \pm}=\frac{\left|t_{\uparrow \pm}\right|^{2}}{\epsilon_{\uparrow} \pm 2 \delta}-\frac{\left|t_{\downarrow \pm}\right|^{2}}{\epsilon_{\downarrow} \pm 2 \delta}, \\
& B_{y}^{ \pm}=\operatorname{Im}\left(t_{\uparrow \pm}^{*} t_{\downarrow \pm}\right)\left(\frac{1}{\epsilon_{\uparrow} \pm 2 \delta}+\frac{1}{\epsilon_{\downarrow} \pm 2 \delta}\right), \\
& B_{z}^{ \pm}=-\operatorname{Re}\left(t_{\uparrow \pm}^{*} t_{\downarrow \pm}\right)\left(\frac{1}{\epsilon_{\uparrow} \pm 2 \delta}+\frac{1}{\epsilon_{\downarrow} \pm 2 \delta}\right),
\end{aligned}
$$

$t_{\sigma \pm}=t_{\sigma l} \pm t_{\sigma r} / i$, and $\delta$ is the splitting due to the overlap of the MBSs closest to the dot on the right $\gamma_{r}$ and left $\gamma_{l}$ topological section. 
Let us consider the case when the ends of the topological sections are sufficiently far apart, $\delta=0$, for which

$$
\begin{aligned}
& \frac{B_{0}^{ \pm}}{t_{0}} \approx \frac{1}{\bar{\epsilon}_{\uparrow}}\left\{e^{-\kappa^{t} \ell} \cos \left[2 k_{\mathrm{SO}} \ell+\pi / 4\right]\right. \\
& \left. \pm e^{\kappa^{t}(\ell-d)} \cos \left[2 k_{\mathrm{SO}}(\ell-d)-\pi / 4\right]\right\}^{2} \\
& +\frac{1}{\bar{\epsilon}_{\downarrow}}\left\{e^{-\kappa^{t} \ell} \cos \left[2 k_{\mathrm{SO}} \ell-\pi / 4\right]\right. \\
& \left.\mp e^{\kappa^{t}(\ell-d)} \cos \left[2 k_{\mathrm{SO}}(\ell-d)+\pi / 4\right]\right\}^{2} \text {, } \\
& \frac{B_{x}^{ \pm}}{t_{0}} \approx \frac{1}{\bar{\epsilon}_{\uparrow}}\left\{e^{-\kappa^{t} \ell} \cos \left[2 k_{\mathrm{SO}} \ell+\pi / 4\right]\right. \\
& \left. \pm e^{\kappa^{t}(\ell-d)} \cos \left[2 k_{\mathrm{SO}}(\ell-d)-\pi / 4\right]\right\}^{2} \\
& -\frac{1}{\bar{\epsilon}_{\downarrow}}\left\{e^{-\kappa^{t} \ell} \cos \left[2 k_{\mathrm{SO}} \ell-\pi / 4\right]\right. \\
& \left.\mp e^{\kappa^{t}(\ell-d)} \cos \left[2 k_{\mathrm{SO}}(\ell-d)+\pi / 4\right]\right\}^{2} \text {, } \\
& \frac{B_{y}^{ \pm}}{t_{0}} \approx \frac{\bar{\epsilon}_{\uparrow}+\bar{\epsilon}_{\downarrow}}{2 \bar{\epsilon}_{\uparrow} \bar{\epsilon}_{\downarrow}}\left\{e^{-2 \kappa^{t} \ell} \cos \left(4 k_{\mathrm{SO}} \ell\right)\right. \\
& -e^{-2 \kappa^{t}(d-\ell)} \cos \left[4 k_{\mathrm{SO}}(d-\ell)\right] \\
& \left. \pm e^{-\kappa^{t} d} \sin \left[2 k_{\mathrm{SO}}(2 \ell-d)\right]\right\}, \quad B_{z}^{ \pm}=0,
\end{aligned}
$$

where the approximations assume $k_{\mathrm{SO}} \gg \kappa_{1}^{t}, \kappa^{t}$ with $\bar{\epsilon}_{\sigma}=$ $\epsilon_{\sigma} / t_{0} \gg 1$ for the perturbative Schrieffer-Wolff transformation to remain valid. Performing the following consecutive operations brings the system [Fig. 1(b)] back to itself: mirror operation in the $y z$ plane, time reversal, and a $\pi$ rotation around the $y$ axis. These operations take $B_{z}^{ \pm} \rightarrow-B_{z}^{ \pm}$, while the other components are invariant. Therefore, $B_{z}^{ \pm}$, i.e., the effective magnetic field along the spin-orbit axis, must be identically zero even for finite overlap of the MBSs, $\delta \neq 0$. As $B_{x}^{+} \neq B_{x}^{-}$and $B_{y}^{+} \neq B_{y}^{-}$, these components are sensitive to the occupancy of the nonlocal fermion. Notice, however, when the quantum dot is far away from one end, e.g., $d \rightarrow \infty$, the effective magnetic field is insensitive to this quantity, $B_{x}^{+}=B_{x}^{-}, B_{y}^{+}=B_{y}^{-}$, and $B_{0}^{+}=B_{0}^{-}$as one may expect.

When the ends of the topological sections are equidistant to the center of the dot, $d=2 \ell$, the components of the effective magnetic field simplify to

$$
\begin{aligned}
& \frac{B_{0}^{ \pm}}{t_{0}}= \pm \frac{B_{x}^{ \pm}}{t_{0}} \approx 8 \frac{\left[1 \mp \sin \left(4 k_{\mathrm{SO}} \ell\right)\right] e^{-2 \kappa^{t} \ell}}{\left(\bar{\epsilon}_{\uparrow}+\bar{\epsilon}_{\downarrow}\right) \pm\left(\bar{\epsilon}_{\uparrow}-\bar{\epsilon}_{\downarrow}\right)}, \\
& B_{y}^{ \pm}=B_{z}^{ \pm}=0 .
\end{aligned}
$$

One may show the system is invariant upon inversion centered at the dot followed by a $\pi$ rotation around the $x$ axis wherein $B_{y}^{ \pm} \rightarrow-B_{y}^{ \pm}$and therefore must be zero. In Fig. 4 (upper panel), we plot the fields $B_{x}^{ \pm}$as a function of $k_{\mathrm{SO}} \ell$. As expected, we see that the component of the effective magnetic field along the axis of the applied magnetic field oscillates with period $\lambda_{\text {So }} / 4$ and changes according to the occupation of the nonlocal fermion.

If the center of the quantum dot is placed slightly asymmetrically, on the scale of the spin-orbit length, between the ends of the topological sections, the effective magnetic field acquires a finite component along the $y$ axis. We plot $B_{x}^{ \pm}$and $B_{y}^{ \pm}$for this geometry in Fig. 4 (middle panel) as a function

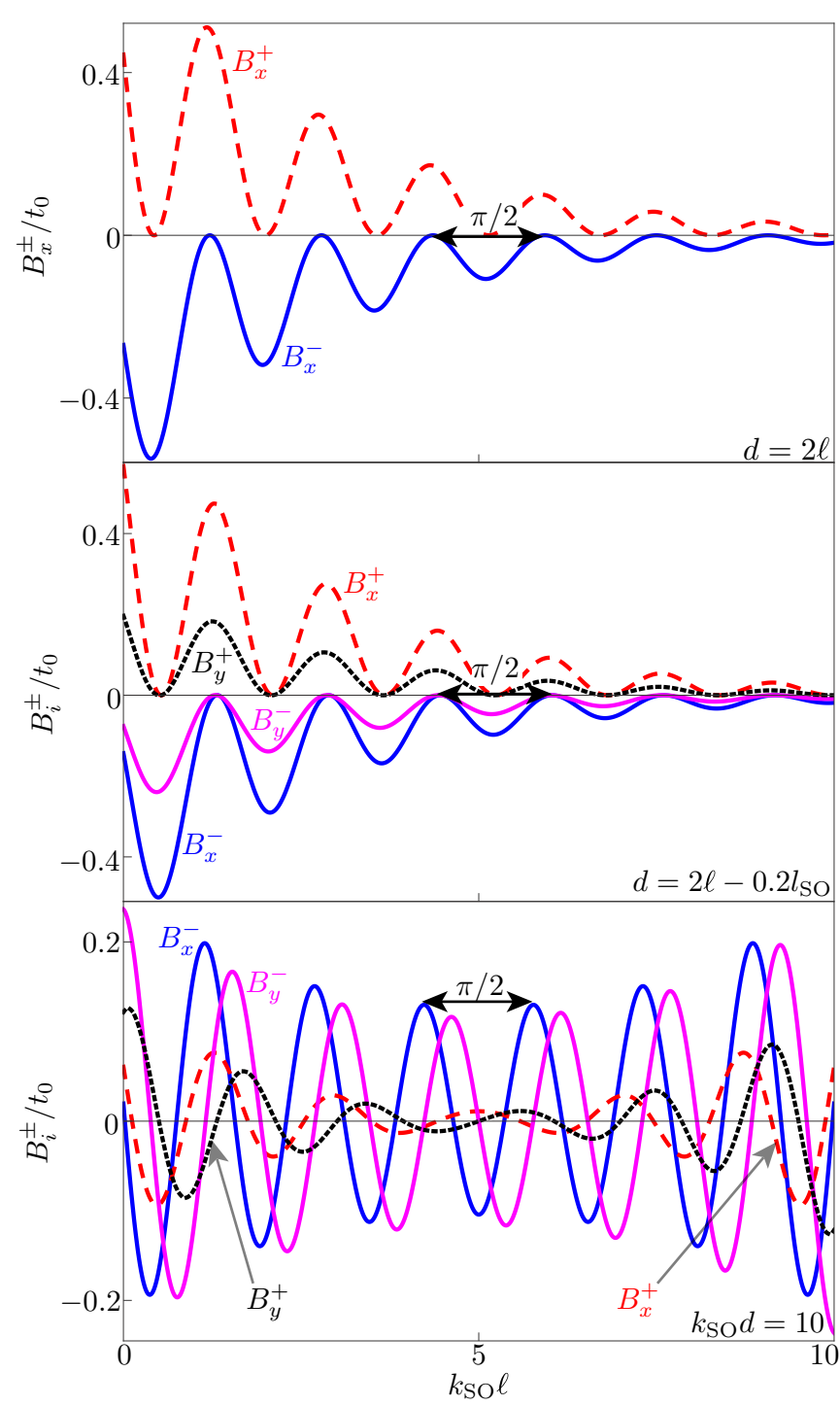

FIG. 4. Effective magnetic field $B_{i}^{ \pm}$induced on a small dot situated (upper panel) equidistant to two MBSs $d=2 \ell$, (middle panel) slightly asymmetrically to the two MBSs $d=2 \ell-0.2 l_{\text {so }}$, and (lower panel) in the case when one fixes the distance between between MBSs $k_{\mathrm{SO}} d=10$, as a function of the distance $k_{\mathrm{SO}} \ell$ between the dot and topological sections for $k_{\mathrm{SO}} L=0.1, \kappa^{t} / k_{\mathrm{SO}}=10 \sqrt{3}$, $\epsilon_{\uparrow}=9 t_{0}$, and $\epsilon_{\downarrow}=11 t_{0}$. Upper panel: The components $B_{x}^{ \pm}$oscillate with period $\lambda_{\text {So }} / 4$ while the other components are zero. Middle panel: $B_{x}^{ \pm}$and $B_{y}^{ \pm}$oscillate with period $\lambda_{\mathrm{SO}} / 4, B_{z}^{ \pm}=0$. Lower panel: $B_{x}^{ \pm}$ $\left(B_{y}^{ \pm}\right)$is a symmetric (antisymmetric) function of $\ell$ around the middle of the nontopological section $k_{\mathrm{SO}} \ell=5$. Both components oscillate with period $\lambda_{\mathrm{SO}} / 4$.

of $k_{\mathrm{SO}} \ell$. The component of the effective magnetic field along the $x$ axis is largely unchanged while the component along $y$ also oscillates with period $\lambda_{\mathrm{so}} / 4$ but smaller amplitude. Furthermore, the local minima and maxima of $B_{x}^{ \pm}$and $B_{y}^{ \pm}$are shifted with respect to each other.

Fixing the distance between two topological sections, we plot the effective magnetic field as a function of distance between the dot and the right topological section in the lower panel of Fig. 4 for the same values as the previous panels. Here $B_{x}^{ \pm}$and $B_{y}^{ \pm}$both oscillate with period $\lambda_{\mathrm{SO}} / 4$ and $B_{y}^{ \pm}=0$ at 
the center of the nontopological section $k_{\mathrm{SO}} \ell=5$ as expected. Again making use of inversion centered in the middle of the dot and a $\pi$ rotation around the $x$ axis, the distance between the dot and the left fermion $(d-\ell)$ is exchanged with the right fermion $(\ell)$ while the remainder of the geometry is invariant. Because of the transformations of the effective magnetic field under this symmetry, we expect $B_{x}^{ \pm}\left(B_{y}^{ \pm}\right)$to be symmetric (antisymmetric) about $\ell=d / 2$, which is readily observed [Fig. 4 (lower panel)].

Large dot-As, in this regime, analytical expressions are too involved to be displayed, we plot directly the effective magnetic field induced on the large dot due to coupling to the MBS states. Analogous to the small $\operatorname{dot}, B_{i}^{ \pm}$is plotted in the upper, middle, and lower panels of Fig. 5 when the left and right MBSs are equidistant from the quantum dot $(d=2 \ell)$, when the quantum dot is placed slightly asymmetrically between the MBSs $\left(d=2 \ell-0.2 l_{\mathrm{SO}}\right)$, and fixing the distance between between the MBSs $\left(k_{\mathrm{SO}} d=20\right)$, respectively. Because the same symmetry arguments can be made, the large dot effective magnetic field is similar to the small dot with the important difference that the oscillations, as a function of $k_{\mathrm{SO}} \ell$, are oscillating with the period $\lambda_{\mathrm{SO}} / 2$ rather than $\lambda_{\mathrm{SO}} / 4$. In particular, when $d=2 \ell$, only $B_{x}^{+} \neq B_{x}^{-}$is finite; when $d=2 \ell-0.2 l_{\mathrm{SO}}$, both $B_{x}^{+} \neq B_{x}^{-}$and $B_{y}^{+} \neq B_{y}^{-}$; and fixing $k_{\mathrm{SO}} d=20, B_{x}^{ \pm}$is symmetric about $k_{\mathrm{SO}} \ell=10$ and $B_{y}^{ \pm}$is antisymmetric about $k_{\mathrm{SO}} \ell=10$.

Experimentally, the tunneling must be less than the finite size energy spacing of the dot, $t_{\sigma}<\hbar \omega_{0}$, which is approximately $50 \mu \mathrm{eV}$ in typical experiments. For the small dot, this corresponds to $t_{0} \approx 50 \mu \mathrm{eV}$ and a maximum effective splitting of about $20 \mu \mathrm{eV}$ or $200 \mathrm{mK}$. Similarly, for the large dot, this corresponds to $t_{0} \approx 3 \mathrm{meV}$ and a maximum effective splitting of about $50 \mu \mathrm{eV}$ or $500 \mathrm{mK}$. Both of which are well above typical experimental temperatures of $20 \mathrm{mK}$.

\section{NUMERICAL SIMULATION}

In this section we numerically study effects resulting from the interplay between quantum dot weakly coupled to one or two wires hosting MBSs in a physical system that one can experimentally engineer. As discussed above, it is difficult to determine the exact MBS wave function when the chemical potential of the nontopological section is in the band gap, so we have focused above on the situation when the nontopological section was created only by a slight detuning of the chemical potential from the SOI energy. In contrast to that, the most viable way to terminate the topological section is to deplete a part of the quantum wire. This scenario we can study numerically by using a tight-binding approach to calculate the spin-dependent tunneling between the dot and MBS (Sec. $\mathrm{V} A$ ) and the spin polarization of the dot, which reflects the effective magnetic field, in the presence of two MBS wires (Sec. V B). We confirm that our analytical results capture the main effects such as oscillations of tunneling amplitude as a function of distance.

\section{A. Spin-dependent tunneling}

We consider an $N$-site tight-binding Bogoliubov-deGennes Hamiltonian (see Fig. 6), analogous to our analytical

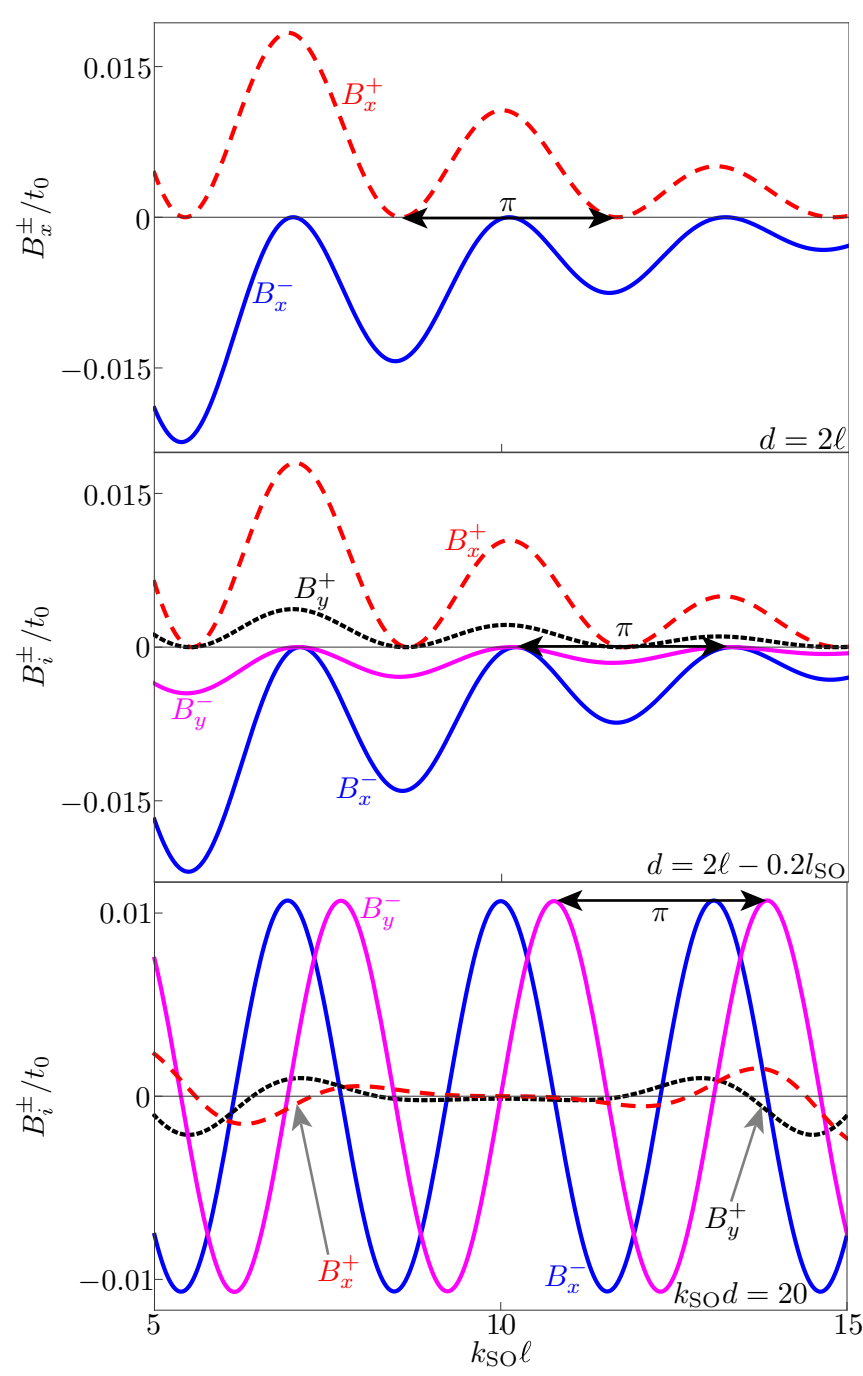

FIG. 5. Effective magnetic field $B_{i}^{ \pm}$induced on a large dot (upper panel) situated equidistant to two MBSs $d=2 \ell$, (middle panel) slightly asymmetrically to the two MBSs $d=2 \ell-0.2 l_{\text {So }}$, and (lower panel) in the case when one fixes the distance between between MBSs $k_{\mathrm{SO}} d=20$, as a function of the distance $k_{\mathrm{SO}} \ell$ between the dot and topological sections for $k_{\mathrm{SO}} L=10, \kappa^{t} / k_{\mathrm{SO}}=10 \sqrt{3}$, and $\epsilon_{\uparrow}=\epsilon_{\downarrow}=t_{0} / 10$. Upper panel: The components $B_{x}^{ \pm}$oscillate with period $\lambda_{\mathrm{SO}} / 2$ while the other components are zero. Middle panel: $B_{x}^{ \pm}$ and $B_{y}^{ \pm}$oscillate with period $\lambda_{\mathrm{So}} / 2, B_{z}^{ \pm}=0$. Lower panel: $B_{x}^{ \pm}\left(B_{y}^{ \pm}\right)$ is symmetric (antisymmetric) function of $\ell$ around the middle of the nontopological section $k_{\mathrm{SO}} \ell=10$.

model,

$$
\begin{aligned}
H= & \sum_{j=1}^{N-1} \Psi_{j+1}^{\dagger}\left(-t-i \tilde{\alpha} \sigma_{3}\right) \eta_{3} \Psi_{j}+\text { H.c. } \\
& +\sum_{j=1}^{N} \Delta_{Z} \Psi_{j}^{\dagger} \sigma_{1} \eta_{3} \Psi_{j}-\mu_{j} \Psi_{j}^{\dagger} \eta_{3} \Psi_{j}+\Delta_{s, j} \Psi_{j}^{\dagger} \sigma_{2} \eta_{2} \Psi_{j}
\end{aligned}
$$

where we are in the Nambu basis $\Psi_{j}^{\dagger}=\left(\psi_{j \uparrow}^{\dagger}, \psi_{j \downarrow}^{\dagger}, \psi_{j, \uparrow}, \psi_{j, \downarrow}\right)$ and the operator $\psi_{j \sigma}^{\dagger}$ creates a particle of $\operatorname{spin} \sigma$ at site $j$. 


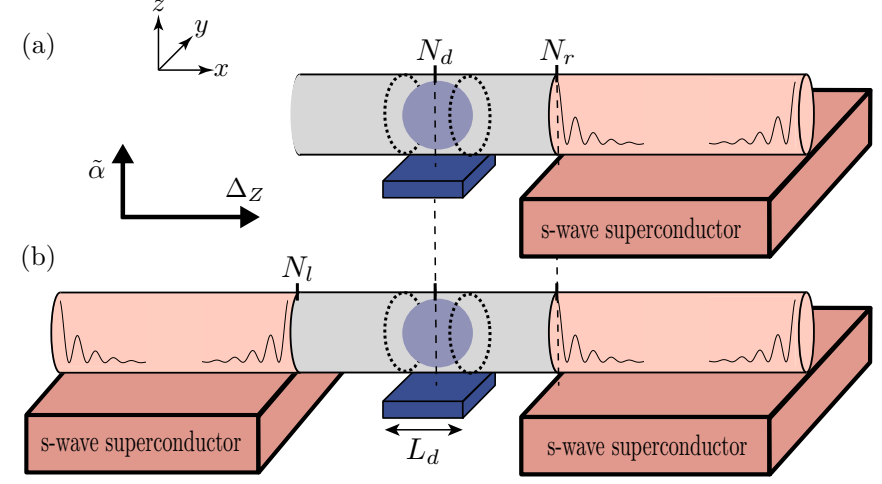

FIG. 6. System setup of the $N$-site tight-binding model. In the upper panel, the topological section (red), defined from site $N_{r}$ to the end of the chain, is realized due to proximity-induced superconductivity. The nontopological section (gray) is driven to the topologically trivial phase by depleting the wire. A quantum dot (blue) of size $L_{d}$ defined by gates is located at $N_{d}<N_{r}$. In the lower panel, the setup is the same with the addition of a second topological section, realized in the same way, from the beginning of the chain to $N_{l}<N_{d}$. In both setups, the magnetic field, inducing a Zeeman splitting $\Delta_{Z}$, is applied along the $x$ axis and the spin-orbit vector, with magnitude $\tilde{\alpha}$, is along the $z$ axis.

The hopping amplitude $t=\hbar^{2} /\left(2 m a^{2}\right)$ is set to 1 and taken as the energy unit and the SOI strength $\tilde{\alpha}$ is fixed to 0.5 for the remainder of the paper. The magnetic field (with Zeeman energy $\Delta_{Z}$ ) is aligned along the $x$ axis and is constant. The SOI vector points along $z$ axis, analogous to the previous section. The chemical potential $\mu_{j}$ is $\mu_{t}$ for $j>N_{r}$ (i.e., the topological section), $\mu_{n}$ for $j<N_{d}-L_{d} / 2$ and $N_{d}+L_{d} / 2<$ $j \leqslant N_{r}$ (the nontopological section excluding the dot), and $\mu_{d}$ for $N_{d}-L_{d} / 2 \leqslant j \leqslant N_{d}+L_{d} / 2$ which defines the quantum dot. The superconducting pairing is zero in the nontopological section $\Delta_{s, j}=0$, for $j \leqslant N_{r}$ and $\Delta_{s}$ otherwise.

We take a wire of length $N=350$ lattice sites. Sites beyond $N_{r}=200$ are driven into the topological phase by taking $\Delta_{s}=0.06, \Delta_{Z}=0.12, \mu_{t}=2$. In the nontopological section of the wire the chemical potential is $\mu_{n}=2.27$ except on the dot, which is of size $L_{d}=31$, where $\mu_{d}=2.245$. Upon diagonalizing the Hamiltonian, we find the position-dependent wave functions and corresponding eigenenergies which are plotted in Fig. 7. The dot wave function, centered at $N_{d}=50$, is Gaussian-like with oscillations at $\lambda_{\mathrm{SO}} / 2=\pi / \tilde{\alpha} \approx 2 \pi$ which correspond to roughly half the spin-orbit wavelength. There are two MBSs, one at each end of the topological section. Within the topological section, the MBS oscillates with period $\lambda_{\text {so }} / 2$. On the left side, the MBS wave function "leaks" into the normal section and has oscillations, also given by the SOI and a smaller relative amplitude proportional to the magnetic field as expected from the analytics [see Eq. (A13)]. It is precisely this leakage and oscillations that results in a position dependent tunneling, and subsequent magnetic field, that we discuss below. In the spectrum, there are two zero energy modes in the center of the plot (see the insert in Fig. 7) corresponding to the MBSs at the ends of the wire. The next two lowest lying energies above zero energy are the spin up and down, nearly degenerate states on the dot. Because the dot size is much

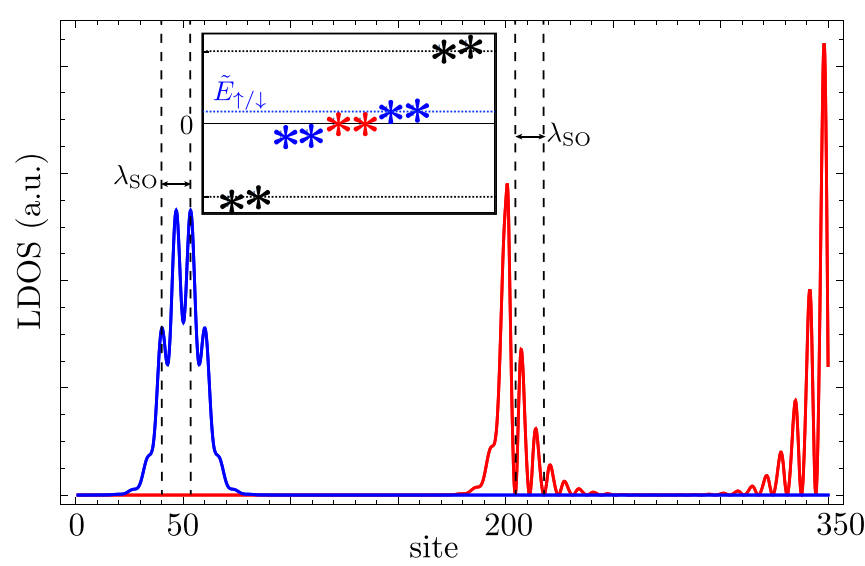

FIG. 7. Local density of states (LDOS) of the wire with one topological section [Fig. 6(a)]. The lowest quantum dot level (blue) is centered at $N_{d}=50$ and of the size $L_{d}=31$ while the MBS wave functions (red) are peaked roughly at the beginning $\left(N_{r}=200\right)$ and at the end $(N=350)$ of the topological section. Here $\Delta_{s}=0.06, \Delta_{Z}=$ $0.12, \mu_{n}=2.27, \mu_{t}=2$, and $\mu_{d}=2.245$. Inset: Energy spectrum indicates two MBS levels (red) at zero energy, two quantum dot levels (blue) with energies at $\tilde{E}_{\uparrow} \approx \tilde{E}_{\downarrow}$, and the next lowest energy levels of the dot (black).

larger than the spin-orbit length, the magnetic field on the dot is exponentially suppressed and the Zeeman splitting on the dot is nearly zero [44].

To extract the spin-dependent tunneling amplitude $\tilde{t}_{\sigma}$ with $\sigma=\uparrow, \downarrow$, we model the dot-MBS system as two weakly coupled levels. Here, because we do not have access to the quantization axis of the dot, $\sigma$ labels the two dot levels. If MBSs leak into the dot, the dot level is shifted from $\tilde{\epsilon}_{\sigma}$ to $\tilde{E}_{\sigma}=\sqrt{\tilde{\epsilon}_{\sigma}^{2}+2 \tilde{t}_{\sigma}^{2}}$ where $\tilde{\epsilon}_{\sigma}$ is the energy of the spin $\sigma$ level when the dot is far from the MBS. Thus, we can extract the spin-dependent coupling $\left|\tilde{t}_{\sigma}\right|=\sqrt{\left(\tilde{E}_{\sigma}^{2}-\tilde{\epsilon}_{\sigma}^{2}\right) / 2}$, which we plot as a function of distance between the dot and MBS in Fig. 8. As expected, the tunneling amplitude $\left|\tilde{t}_{\sigma}\right|$ decreases exponentially and oscillates with period $\lambda_{\mathrm{SO}} / 2$ as the distance between the dot and MBS increases. Furthermore, the tunneling amplitudes are offset from each other by a phase $\pi / 2$, in agreement with

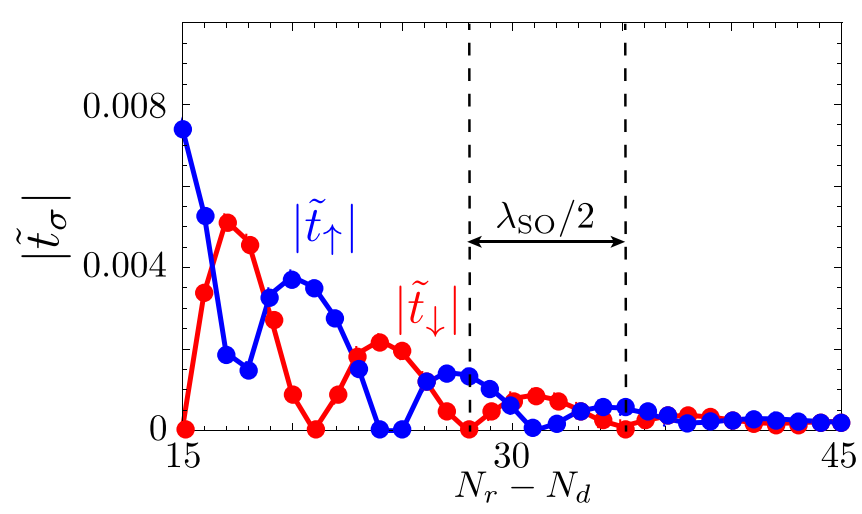

FIG. 8. Tunneling amplitudes $\left|\tilde{t}_{\uparrow}\right|$ and $\left|\tilde{t}_{\downarrow}\right|$ between the quantum dot and MBS [Fig. 6(a)] as a function of the distance between the position of the dot and the end of the topological section of the wire. All parameters are the same as in Fig. 7. 


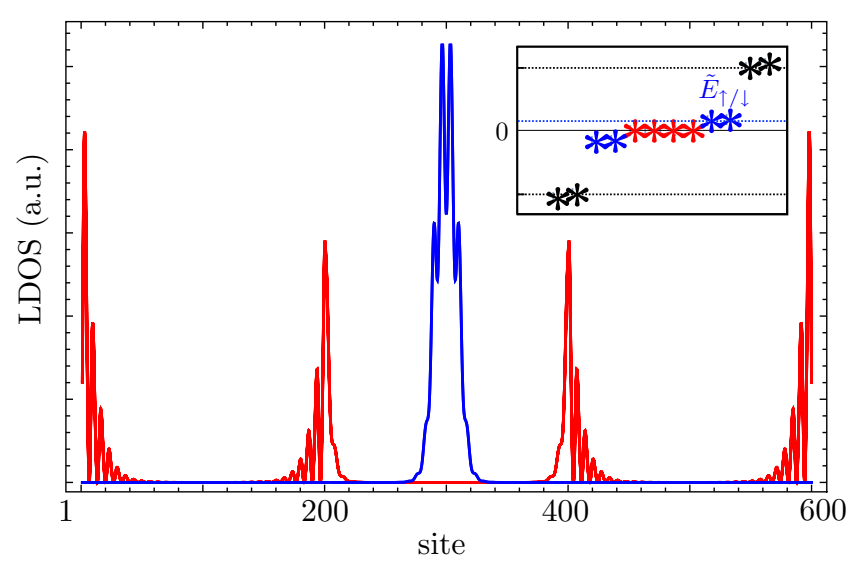

FIG. 9. Local density of states of a chain with two identical topological sections [Fig. 6(b)] between $N=1$ and $N_{l}=200$ and between $N_{r}=400$ and the end of the chain $(N=600)$. The quantum dot wave function (blue) is centered around the dot position $N_{d}=300$ and there are four MBSs at the interfaces of the topological and nontopological sections. Inset: Energy spectrum indicates four zero energy states corresponding to the MBSs (red), the lowest energy dot levels (blue), and second lowest dot levels (black). The system parameters are the same as in Fig. 7.

the analytics. Because we are only probing the change in energy of the dot level, we can only determine the magnitude of the spin-dependent tunneling; we expect that $\tilde{t}_{\sigma}$ to oscillate with periodicity $\lambda_{\text {SO }}$.

\section{B. Effective magnetic field}

Next, we extend our model [see Eq. (20)] and add an additional topological section to the left of the dot [see Fig. 6(b)]. The site-dependent parameters are redefined as follows: The chemical potential is $\mu_{t}$ for $j \leqslant N_{l}$ and $j>N_{r}$ where $N_{l}$ now defines the end of the second topological section, $\mu_{n}$ for $N_{l}<j<N_{d}-L_{d} / 2$ and $N_{d}+L_{d} / 2<j \leqslant N_{r}$, and $\mu_{d}$ for $N_{d}-L_{d} / 2 \leqslant j \leqslant N_{d}+L_{d} / 2$. The superconducting pairing is zero, $\Delta_{s, j}=0$, for $N_{l}<j \leqslant N_{r}$ and $\Delta_{s}$ otherwise.

We now take $N=600, N_{d}=300$ with $N_{r}$ and $N_{l}$ free to vary. All other parameters are left unchanged. The sites with $j \leqslant N_{l}$ and $j>N_{r}$ are in the topological regime. Plotting the wave functions (see Fig. 9), we see, accordingly, that there are indeed four MBS states at the four interfaces of the topological with nontopological sections, all of which sit at zero energy. The characteristics of MBS and dot level wave functions (delay lengths and period of oscillations) are the same as in the previous subsection.

To extract the effective magnetic field on the dot, we calculate the spin of the dot by summing the expectation of the spin operator, $\hat{S}_{x}=\sigma_{1} \eta_{3}, \hat{S}_{y}=\sigma_{2}$, and $\hat{S}_{z}=\sigma_{3} \eta_{3}$, at all sites where the dot level has finite weight. In Fig. 10 we present the spin on the dot, $\tilde{S}_{i}=\sum_{j} \tilde{Y}_{j}^{\dagger} \hat{S}_{i} \tilde{Y}_{j}$ (measured in units of $\hbar / 2$ ) with $\tilde{Y}_{j}$ the dot wave function at site $j$, as a function of the distance between the dot and MBSs. Analogous to the previous section, we have considered nearly symmetrically placed topological sections so that the MBS on the left and right are equidistant to the dot up to one lattice constant, i.e., $N_{d}-N_{l}-1=N_{r}-N_{d}-1$. Similar to the analytic results,

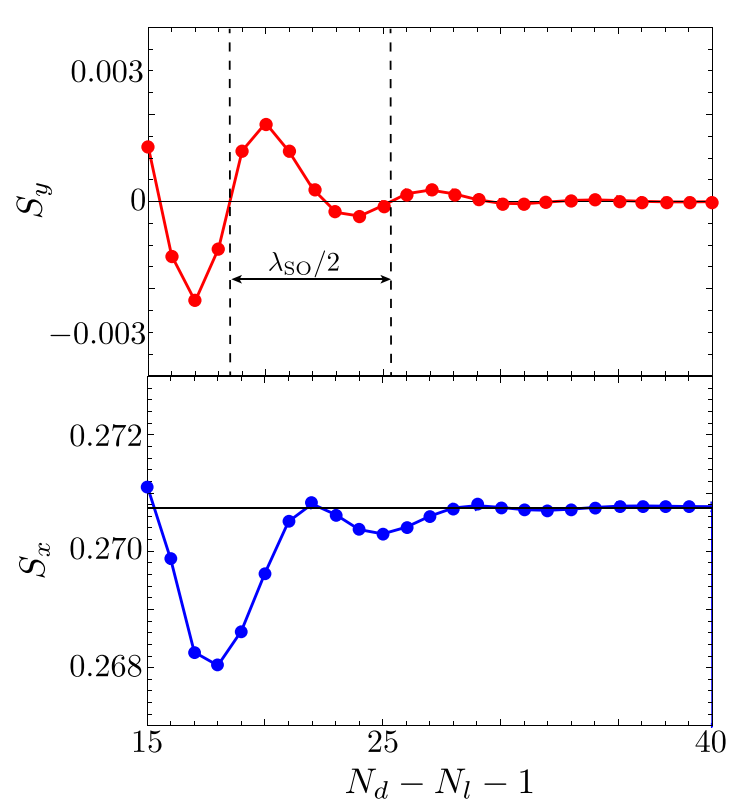

FIG. 10. The spin components of the lowest quantum dot level along the $y$ and $x$ axes, respectively, as a function of the distance between the dot and end of the left and right topological sections, which are kept equidistant to the dot up to one lattice constant, $N_{d}-N_{l}-1=N_{r}-N_{d}-1$. Both components oscillate with period $\lambda_{\mathrm{SO}} / 2$, and depend exponentially on the distance between the dot and the MBSs. The spin projection on the $y$ axis goes to zero when the dot is far from MBSs while the $x$ component saturates at the value determined by the external magnetic field (black solid line around $\approx 0.271$ ). We note that the component $\tilde{S}_{z}$ is always zero due to the symmetry of the problem.

we see oscillations in spin on the dot with period $\lambda_{\mathrm{SO}} / 2$ along the $x$ and $y$ axes while the spin along the SOI axis is exactly zero. The offset of $\tilde{S}_{x}$ in Fig. 10 is the result of a residual magnetic field coming from the applied external Zeeman field along the $x$ direction (see Appendix $\mathrm{C}$ for details). We note that in Fig. 10, because $L_{d}$ is odd and $N_{r}-N_{l}$ is even, the dot is closer to the left topological section than to the right topological section by one lattice constant. As a result, $\tilde{S}_{y} \neq 0$, which is consistent with our analytical predictions. If the dot is placed equidistantly between the two topological sections, $\tilde{S}_{y}$ is zero.

In contrast to the analytic results, there are two important differences in the tight-binding calculation: (1) we are unable to account for many-body interactions and therefore cannot differentiate between a filled and unfilled nonlocal fermion nor can we include a finite Coulomb interaction on the dot; and (2) the difference in physical realizations of the topological-nontoplogical junctions. Despite these differences between the models, we find a striking similarity in the spindependent tunneling and effective magnetic field. We attribute this to the equality of the symmetries in the analytic and numerical models. Therefore, we expect any MBS-quantum dot system that obeys such symmetries, regardless of how the topological and nontopological regimes are realized, to display similar behavior of the spin-dependent tunneling and effective magnetic field. 


\section{CONCLUSIONS AND OUTLOOK}

We have shown analytically and numerically that the tunneling amplitudes between MBSs and a nearby quantum dot are spin dependent and also depend on the distance between the dot and topological section hosting MBSs. Generally, the spin up and down tunneling amplitudes are oscillating on the scale of the SOI length. In particular, depending on this distance, the tunnel amplitudes can be made to be completely spin polarized. Analogously, the effective magnetic field induced on a quantum dot by two MBSs depends on the distance between topological sections and quantum dot and, unlike the tunneling, on the occupancy of the nonlocal fermion formed from the MBSs. Any phenomenological Hamiltonian between MBSs and quantum dots must include a spin dependence in the tunneling in order to be applied to Rashba quantum wires. Furthermore, this warrants a reconsideration of the assumption of spin-independent tunneling between quantum dots and MBS for a broad range of parameters and systems.

When the SOI length is large and the boundary between topological and nontopological sections or quantum dot are mobile, one could use the relative positions of the two as a way to fine tune the spin dependence of the tunneling. Alternatively, if the relative positions are fixed or the SOI length is smaller than the experimental precision, the spin dependence cannot be adjusted and thus may be a source of error. This is especially problematic when combining braiding and readout of MBS qubits using quantum dots [19]. That is, after a braiding operation, the distance between the MBS and quantum dot must be brought back to a precise position. If not, the qubit readout must be recalibrated. Additionally, the quantum dot may be at a distance where the tunneling to one of the levels is small. In MBS-spin qubit quantum computation schemes [19], the operation speed scales as $\epsilon_{0} \hbar / \operatorname{Im}\left(t_{\uparrow}^{*} t_{\downarrow}\right)$. Whereas in MBS-quantum dot computation schemes, when only a single level of the dot is accessible [18,21], the operation speed is limited by the single-level coupling to the MBS, e.g., if the spin up level is closest to chemical potential then the operation speed scales as $\epsilon_{\uparrow} \hbar /\left|t_{\uparrow}\right|^{2}$. In both cases, a small coupling can have a significant detrimental effect on the operation speed.

In lieu of a quantum wire, one could use a magnetic atomic chain deposited on the surface of a superconductor which has been theoretically [49-52] and experimentally [7,8] shown to support MBS end states. The local helical magnetic field of the helical chain is equivalent to the SOI and homogeneous magnetic field. An auxiliary two level atom coupled to the ends of two such chains, analogous to the dot in our quantum wires setup, could be used to probe these MBSs. We foresee two mechanisms by which the auxiliary atom can couple to the chain: the overlap of wave function of the orbital levels in the auxiliary atom with either the hybridized conduction bands in the atomic chain or with the bulk quasiparticles in the superconductor. When there is a direct tunneling between the orbital levels of the chain and the dot, we expect only the magnitude of the tunneling between MBSs and levels in the auxiliary atom to vary as a function of the distance between the two because there is no analog of the SOI or magnetic field outside the chain. If there is a SOI in the superconductor, the spin dependence of the tunneling could depend on the distance between the chain and auxiliary atoms, analogous to the role of the SOI in the quantum wire. We also note that instead of a quantum dot levels, alternatively, one can also use finite-energy bound states inside the superconducting gap, for example, occurring due to change in the direction of the SOI vector [53]. Again, we expect that the overlap between such bound states and MBSs decays exponentially with the distance as well as oscillates on the scale set by the SOI length.

\section{ACKNOWLEDGMENTS}

We acknowledge helpful discussions with Constantin Schrade, Pawel Szumniak, and Marcel Serina. This work was supported by the Swiss National Science Foundation and NCCR QSIT.

\section{APPENDIX A: MAJORANA BOUND STATE WAVE FUNCTIONS}

\section{Small deviations of the chemical potential from the SOI energy}

In this Appendix we derive the MBS wave function given in the main text [see Eq. (4)]. The general wave functions on the left (nontopological section) and right (topological section) side are written as

$$
\Phi^{t}=A^{t} \Phi_{1}^{t}+B^{t} \Phi_{2}^{t}+C^{t} \Phi_{3}^{t}+D^{t} \Phi_{4}^{t}, \quad \Phi^{n}=A^{n} \Phi_{1}^{n}+B^{n} \Phi_{2}^{n}+C^{n} \Phi_{3}^{n}+D^{n} \Phi_{4}^{n},
$$

respectively, where the coefficients must be real if the solutions are MBSs. To satisfy continuity, the coefficients must satisfy the equations

$$
A^{t}+C^{t}=-\cos \varphi_{n} A^{n}-\sin \varphi_{n} B^{n}+C^{n}, \quad A^{t}+C^{t}=\cos \varphi_{n} A^{n}+\sin \varphi_{n} B^{n}+C^{n},
$$

which we have obtained by taking the imaginary part of the first component and the real part of the second component, respectively. This implies that $\cos \varphi_{n} A^{n}+\sin \varphi_{n} B^{n}=0$ and $C^{n}=A^{t}+C^{t}$. Using the former condition, differentiability of the solutions requires

$$
\begin{gathered}
-\left(\kappa_{1}^{n}-\kappa_{2}^{n}\right) \cos \varphi_{n} A^{n}+\kappa^{n} C^{n}+2 k_{\mathrm{SO}} D^{n}=-\kappa_{1}^{t} A^{t}-\kappa^{t} C^{t}+2 k_{\mathrm{SO}} D^{t}, \\
\left(\kappa_{1}^{n}-\kappa_{2}^{n}\right) \cos \varphi_{n} A^{n}+\kappa^{n} C^{n}+2 k_{\mathrm{SO}} D^{n}=-\kappa_{1}^{t} A^{t}-\kappa^{t} C^{t}+2 k_{\mathrm{SO}} D^{t} .
\end{gathered}
$$

Therefore, because $\kappa_{1}^{n} \neq \kappa_{2}^{n}$ and $\varphi_{n} \neq 0, A^{n}=B^{n}=0$. Continuity further implies, after taking the real and imaginary parts of the first and second components, respectively,

$$
D^{n}=B^{t}+D^{t}, \quad D^{n}=-B^{t}+D^{t},
$$


so that $B^{t}=0$ and $D^{n}=D^{t}$. With Eq. (A2), one may show that $C^{t}=-A^{t}\left(\kappa^{n}+\kappa_{1}^{t}\right) /\left(\kappa^{n}+\kappa^{t}\right)$ and $C^{n}=A^{t}\left(\kappa^{t}-\kappa_{1}^{t}\right) /\left(\kappa^{n}+\kappa^{t}\right)$. Finally, invoking differentiability, one finds

$$
-2 k_{\mathrm{SO}} C^{n}+D^{t} \kappa^{n}=-\kappa_{\mathrm{SO}} C^{t}-\kappa^{t} D^{t},
$$

and $D^{t}=2 k_{\mathrm{SO}}\left(C^{n}-C^{t}\right) /\left(\kappa^{n}+\kappa^{t}\right)=2 k_{\mathrm{SO}} /\left(\kappa^{n}+\kappa^{t}\right)$. Thus, we recover Eq. (4) in the main part.

\section{Chemical potential is in the band gap (depletion)}

When the nontopological section is characterized by the chemical potential being inside the band gap such that this section is depleted, the wave functions are different than ones found above. In order to find these wave functions, we assume that $\mu_{l}$ is much larger than the SOI energy, the superconducting gap, and the magnetic field (the last two are put to zero in the nontopological section). In this case, we find that the eigenstates of the Hamiltonian are

$$
\Psi_{k}^{ \pm}=\psi^{ \pm} e^{i k x}, \quad \mathcal{X}_{k}^{ \pm}=\chi^{ \pm} e^{i k x},
$$

with energies $\left(k^{2} / 2 m-\mu_{l}\right) \pm \alpha k$ and $-\left[\left(k^{2} / 2 m-\mu_{l}\right) \pm \alpha k\right]$, respectively, where $\left(\psi^{+}\right)^{T}=(1,0,0,0),\left(\psi^{-}\right)^{T}=(0,1,0,0)$, $\left(\chi^{+}\right)^{T}=(0,0,1,0)$, and $\left(\chi^{-}\right)^{T}=(0,0,0,1)$. The zero energy solutions of Eq. (A5) require $k \equiv k^{\mp}=\mp k_{\text {so }}-i \kappa_{F}$, where $\kappa_{F}=\sqrt{2 m \mu_{l}} / \hbar$ and we have chosen solutions that vanish as $x \rightarrow-\infty$. It is easiest to match the topological section by finding linear superpositions that are MBSs,

$$
\begin{aligned}
& \Phi_{1}^{n}=-i \Psi^{+}+\Psi^{-}+i \mathcal{X}^{+}+\mathcal{X}^{-}, \quad \Phi_{2}^{n}=\Psi^{+}-i \Psi^{-}+\mathcal{X}^{+}+i \mathcal{X}^{-}, \quad \Phi_{3}^{n}=i \Psi^{+}+\Psi^{-}-i \mathcal{X}^{+}+\mathcal{X}^{-}, \\
& \Phi_{4}^{n}=\Psi^{-}+i \Psi^{-}+\mathcal{X}^{+}-i \mathcal{X}^{-},
\end{aligned}
$$

where $\Psi^{ \pm}=\Psi_{\mp k}^{ \pm}$and $\mathcal{X}^{ \pm}=\mathcal{X}_{\mp k}^{ \pm}$.

Because these are now of the form of the MBSs in the nontopological section, it is straightforward to find conditions for continuity of the MBSs at the boundary which are $A^{n}=0, C^{n}=C^{t}+A^{t}, B^{t}=B^{n}$, and $D^{t}=D^{n}$, where the coefficients have been defined analogous to Eq. (A1). Upon solving the conditions for differentiability, we find $B^{t}=0$,

$$
C^{t}=-A^{t} \frac{3 k_{\mathrm{SO}}^{2}+\kappa_{F}^{2}+\kappa_{F} \kappa^{t}+\kappa_{F} \kappa_{1}^{t}+\kappa^{t} \kappa_{1}^{t}}{9 k_{\mathrm{SO}}^{2}+\left(\kappa_{F}+\kappa^{t}\right)^{2}}, \quad D^{t}=A^{t} \frac{k_{\mathrm{SO}}\left(\kappa_{F}-\kappa^{t}+3 \kappa_{1}^{t}\right)}{9 k_{\mathrm{SO}}^{2}+\left(\kappa_{F}+\kappa^{t}\right)^{2}} .
$$

Therefore, the MBS wave function is given by

$$
\begin{gathered}
\Phi^{t}=A^{t}\left(\Phi_{1}^{t}-\frac{3 k_{\mathrm{SO}}^{2}+\kappa_{F}^{2}+\kappa_{F} \kappa^{t}+\kappa_{F} \kappa_{1}^{t}+\kappa^{t} \kappa_{1}^{t}}{9 k_{\mathrm{SO}}^{2}+\left(\kappa_{F}+\kappa^{t}\right)^{2}} \Phi_{3}^{t}+\frac{k_{\mathrm{SO}}\left(\kappa_{F}-\kappa^{t}+3 \kappa_{1}^{t}\right)}{9 k_{\mathrm{SO}}^{2}+\left(\kappa_{F}+\kappa^{t}\right)^{2}} \Phi_{4}^{t}\right), \\
\Phi^{n}=A^{t}\left(\frac{6 k_{\mathrm{SO}}^{2}+\left(\kappa^{t}\right)^{2}+\kappa_{F} \kappa^{t}-\kappa_{F} \kappa_{1}^{t}-\kappa^{t} \kappa_{1}^{t}}{9 k_{\mathrm{SO}}^{2}+\left(\kappa_{F}+\kappa^{t}\right)^{2}} \Phi_{3}^{n}+\frac{k_{\mathrm{SO}}\left(\kappa_{F}-\kappa^{t}+3 \kappa_{1}^{t}\right)}{9 k_{\mathrm{SO}}^{2}+\left(\kappa_{F}+\kappa^{t}\right)^{2}} \Phi_{4}^{n}\right) .
\end{gathered}
$$

Adding a magnetic field perturbatively, to first order in the Zeeman energy $\Delta_{Z}$ the energies are unchanged, while the eigenvectors are transformed as

$$
\Psi_{k}^{ \pm} \rightarrow \tilde{\psi}_{k}^{ \pm}=\Psi_{k}^{ \pm} \pm \Delta_{Z} \Psi_{k}^{\mp} / 2 \alpha k, \quad \mathcal{X}_{k}^{ \pm} \rightarrow \tilde{\chi}_{k}^{ \pm}=\mathcal{X}_{k}^{ \pm} \pm \Delta_{Z} \mathcal{X}_{k}^{\mp} / 2 \alpha k .
$$

The zero energy solutions are thus $\tilde{\psi}^{ \pm}=\tilde{\psi}_{\mp k}^{ \pm}$and $\tilde{\chi}^{ \pm}=\tilde{\chi}_{\mp k}^{ \pm}$. It is convenient to define

$$
\tilde{\Psi}^{ \pm}=\tilde{\psi}^{ \pm} \mp \Delta_{Z} \tilde{\psi}^{\mp} / k^{\mp}, \quad \tilde{\mathcal{X}}^{ \pm}=\tilde{\chi}^{ \pm} \pm \Delta_{Z} \tilde{\chi}^{\mp} / k^{ \pm},
$$

so that, to leading order in the Zeeman splitting, $\left.\tilde{\psi}^{ \pm}\right|_{x=\ell}=\psi^{ \pm}$and $\left.\tilde{\chi}^{ \pm}\right|_{x=\ell}=\chi^{ \pm}$. We find the zero energy MBSs analogously,

$$
\begin{aligned}
& \tilde{\Phi}_{1}^{n}=-i \tilde{\Psi}^{+}+\tilde{\Psi}^{-}+i \tilde{\mathcal{X}}^{+}+\tilde{\mathcal{X}}^{-}, \quad \Phi_{2}^{n}=\tilde{\Psi}^{+}-i \tilde{\Psi}^{-}+\tilde{\mathcal{X}}^{+}+i \tilde{\mathcal{X}}^{-}, \quad \Phi_{3}^{n}=i \tilde{\Psi}^{+}+\tilde{\Psi}^{-}-i \tilde{\mathcal{X}}^{+}+\tilde{\mathcal{X}}^{-}, \\
& \Phi_{4}^{n}=\tilde{\Psi}^{-}+i \tilde{\Psi}^{-}+\tilde{\mathcal{X}}^{+}-i \tilde{\mathcal{X}}^{-},
\end{aligned}
$$

or in a more suggestive form

$$
\begin{aligned}
& \bar{\Phi}_{1}^{n}=\left(\begin{array}{c}
-i e^{-i k_{\mathrm{so}}(x-\ell)}+i \mathcal{S}^{+} \\
e^{i k_{\mathrm{so}}(x-\ell)}+\mathcal{S}^{-} \\
i e^{i k_{\mathrm{so}}(x-\ell)}-i \mathcal{S}^{-} \\
e^{-i k_{\mathrm{so}}(x-\ell)}+\mathcal{S}^{+}
\end{array}\right) e^{\kappa_{F}(x-\ell)}, \quad \bar{\Phi}_{2}^{n}=\left(\begin{array}{c}
e^{-i k_{\mathrm{so}}(x-\ell)}+\mathcal{S}^{+} \\
-i e^{i k_{\mathrm{so}}(x-\ell)}+i \mathcal{S}^{-} \\
e^{i k_{\mathrm{so}}(x-\ell)}+\mathcal{S}^{-} \\
i e^{-i k_{\mathrm{so}}(x-\ell)}-i \mathcal{S}^{+}
\end{array}\right) e^{\kappa_{F}(x-\ell)}, \\
& \bar{\Phi}_{3}^{n}=\left(\begin{array}{c}
i e^{-i k_{\mathrm{so}}(x-\ell)}+i \mathcal{S}^{+} \\
e^{i k_{\mathrm{so}}(x-\ell)}-\mathcal{S}^{-} \\
-i e^{i k_{\mathrm{so}}(x-\ell)}-i \mathcal{S}^{-} \\
e^{-i k_{\mathrm{so}}(x-\ell)}-\mathcal{S}^{+}
\end{array}\right) e^{\kappa_{F}(x-\ell)}, \quad \bar{\Phi}_{4}^{n}=\left(\begin{array}{c}
e^{-i k_{\mathrm{so}}(x-\ell)}-\mathcal{S}^{+} \\
i e^{i k_{\mathrm{so}}(x-\ell)}+i \mathcal{S}^{-} \\
e^{i k_{\mathrm{so}}(x-\ell)}-\mathcal{S}^{-} \\
-i e^{-i k_{\mathrm{so}}(x-\ell)}-i \mathcal{S}^{+}
\end{array}\right) e^{\kappa_{F}(x-\ell)},
\end{aligned}
$$


where $\mathcal{S}^{ \pm}=\Delta_{Z} \sin \left[(x-\ell) k_{\mathrm{SO}}\right] / \alpha k^{ \pm}$. Because the MBSs now have contributions from both left and right moving branches in the nontopological section of the wire, $\left|\bar{\Phi}_{i}\right|^{2}$ oscillates with periodicity proportional to $k_{\text {So }}$ and amplitude $\Delta_{Z}$. Therefore, Eq. (A13) suggests, in contrast to the small chemical potential, the probability of the MBS wave function satisfying the boundary conditions also oscillates in the nontopological section. Although we find continuous and differentiable solutions when the magnetic field is zero, the condition of differentiability breaks down for finite magnetic field as alluded to in Sec. II of the main text. We focus on an analytic study in the regime of a small chemical potential and study the large chemical potential regime in Sec. V, where we use a numerical tight-binding approach.

\section{APPENDIX B: EFFECTIVE COUPLING BETWEEN DOT AND MBSS}

In this Appendix we calculate the effective exchange Hamiltonian between the quantum dot levels and MBSs by generalizing the work done in Ref. [19], which calculated the an effective exchange Hamiltonian for spin-independent tunneling amplitudes, for spin-dependent ones $\tilde{t}_{\lambda \sigma}$. Following that reference, we take consider a system of two finite size TSC where $\tilde{\gamma}_{\lambda}$ and $\tilde{\gamma}_{\lambda}^{\prime}$ are the MBSs in the left and right ends, respectively, of wire $\lambda$ where the total Hamiltonian describing this system is defined by

$$
\begin{aligned}
& H=\tilde{H}_{M}+H_{D}+\tilde{H}_{T}, \\
& \tilde{H}_{M}=i \sum_{\lambda} \tilde{\delta}_{\lambda} \tilde{\gamma}_{\lambda}^{\prime} \tilde{\gamma}_{\lambda}, \\
& H_{D}=\sum_{\sigma} \epsilon_{\sigma} d_{\sigma}^{\dagger} d_{\sigma}+U n_{\sigma} n_{\bar{\sigma}} / 2, \\
& \tilde{H}_{T}=\sum_{\sigma, \lambda} d_{\sigma}^{\dagger}\left(i \tilde{t}_{\sigma \lambda}^{\prime} \tilde{\gamma}_{\lambda}^{\prime}+\tilde{t}_{\sigma \lambda} \tilde{\gamma}_{\lambda}\right)+\left(\tilde{t}_{\sigma \lambda}^{*} \tilde{\gamma}_{\lambda}-i t_{\sigma \lambda}^{\prime *} \tilde{\gamma}_{\lambda}^{\prime}\right) d_{\sigma} .
\end{aligned}
$$

Here $\tilde{\delta}_{\lambda}$ is the splitting of the MBSs in TSC $\lambda, U$ is the Coulomb repulsion on the dot, and $\tilde{t}_{\sigma \lambda}^{\prime}\left(\tilde{t}_{\sigma \lambda}\right)$ is the matrix element for an electron with spin $\sigma$ on the dot tunneling to the MBS in the left (right) end of the $\lambda$ th TSC. We rewrite the Majorana bound states as $\tilde{f}_{\lambda}=\left(\tilde{\gamma}_{\lambda}^{\prime}+i \tilde{\gamma}_{\lambda}\right) / 2$ so that $\tilde{f}_{\lambda}^{\dagger} \tilde{f}_{\lambda}=\left(1+i \tilde{\gamma}_{\lambda}^{\prime} \tilde{\gamma}_{\lambda}\right) / 2$ and $i \tilde{\delta}_{\lambda} \tilde{\gamma}_{\lambda}^{\prime} \tilde{\gamma}_{\lambda}=\tilde{\delta}_{\lambda}\left(2 \tilde{f}_{\lambda}^{\dagger} \tilde{f}_{\lambda}-1\right)$. The logical values of the MBS qubit are written in terms of the parity of the left and right wires. Using $\tilde{\gamma}_{\lambda}^{\prime}=\tilde{f}_{\lambda}+\tilde{f}_{\lambda}^{\dagger}$ and $\tilde{\gamma}_{\lambda}=\left(\tilde{f}_{\lambda}-\tilde{f}_{\lambda}^{\dagger}\right) / i$, the tunneling Hamiltonian is transformed into

$$
\begin{aligned}
\tilde{H}_{T} & =\sum_{\sigma \lambda} d_{\sigma}^{\dagger}\left[i \tilde{t}_{\sigma \lambda}\left(\tilde{f}_{\lambda}+\tilde{f}_{\lambda}^{\dagger}\right)-i t_{\sigma \lambda}^{\prime}\left(\tilde{f}_{\lambda}-\tilde{f}_{\lambda}^{\dagger}\right)\right]+\left[-i t_{\sigma \lambda}^{\prime *}\left(\tilde{f}_{\lambda}-\tilde{f}_{\lambda}^{\dagger}\right)-i \tilde{t}_{\sigma \lambda}^{*}\left(\tilde{f}_{\lambda}+\tilde{f}_{\lambda}^{\dagger}\right)\right] d_{\sigma} \\
& =\sum_{\sigma \lambda} i\left(\tilde{t}_{\sigma \lambda}^{*}-\tilde{t}_{\lambda}^{*}\right) \tilde{f}_{\lambda}^{\dagger} d_{\sigma}-i\left(\tilde{t}_{\sigma \lambda}^{\prime *}+\tilde{t}_{\sigma \lambda}^{*}\right) \tilde{f}_{\lambda} d_{\sigma}+i\left(\tilde{t}_{\sigma \lambda}-\tilde{t}_{\lambda}^{\prime}\right) d_{\sigma}^{\dagger} \tilde{f}_{\lambda}+i\left(\tilde{t}_{\sigma \lambda}^{\prime}+\tilde{t}_{\sigma \lambda}\right) d_{\sigma}^{\dagger} \tilde{f}_{\lambda}^{\dagger} \\
& =\sum_{\sigma \lambda} i \tilde{t}_{\sigma \lambda-}^{*} \tilde{f}_{\lambda}^{\dagger} d_{\sigma}-i \tilde{t}_{\sigma \lambda+}^{*} \tilde{f}_{\lambda} d_{\sigma}-i \tilde{t}_{\sigma \lambda-} d_{\sigma}^{\dagger} \tilde{f}_{\lambda}+i \tilde{t}_{\sigma \lambda+} d_{\sigma}^{\dagger} \tilde{f}_{\lambda}^{\dagger}
\end{aligned}
$$

where $\tilde{t}_{\sigma \lambda \pm}=\tilde{t}_{\sigma \lambda}^{\prime} \pm \tilde{t}_{\sigma \lambda}$. Here the term proportional to $\tilde{t}_{\sigma \lambda-}$ and its complex conjugate correspond to normal tunneling while the term proportional to $\tilde{t}_{\sigma \lambda+}$ and its complex conjugate correspond to Andreev reflection. Using a Schrieffer-Wolff transformation $[48,54]$, one may show that the operators $A_{\sigma \lambda}-A_{\sigma \lambda}^{\dagger}$ and $B_{\sigma \lambda}-B_{\sigma \lambda}^{\dagger}$ eliminate the tunneling Hamiltonian $\tilde{H}_{T}=-\sum_{\sigma \lambda}\left[A_{\sigma \lambda}-\right.$ $\left.A_{\sigma \lambda}^{\dagger}+B_{\sigma \lambda}-B_{\sigma \lambda}^{\dagger}, \tilde{H}_{M}+H_{D}\right]$, to first order in $\tilde{t}_{\sigma \lambda \pm}$, where

$$
\begin{aligned}
A_{\sigma \lambda} & =i\left(\tilde{t}_{\sigma \lambda}^{*}-\tilde{t}_{\sigma \lambda}^{*}\right)\left[\frac{1}{\epsilon_{\sigma}-2 \tilde{\delta}_{\lambda}}-\frac{U n_{\bar{\sigma}}}{\left(\epsilon_{\sigma}-2 \tilde{\delta}_{\lambda}\right)\left(\epsilon_{\sigma}+U-2 \tilde{\delta}_{\lambda}\right)}\right] \tilde{f}_{\lambda}^{\dagger} d_{\sigma} \\
& =-i \tilde{t}_{\sigma \lambda-}^{*}\left[\frac{1}{\epsilon_{\sigma}-2 \tilde{\delta}_{\lambda}}-\frac{U n_{\bar{\sigma}}}{\left(\epsilon_{\sigma}-2 \tilde{\delta}_{\lambda}\right)\left(\epsilon_{\sigma}+U-2 \tilde{\delta}_{\lambda}\right)}\right] \tilde{f}_{\lambda}^{\dagger} d_{\sigma}, \\
B_{\sigma \lambda} & =i\left(\tilde{t}_{\sigma \lambda}^{*}+\tilde{t}_{\sigma \lambda}^{\prime *}\right)\left[\frac{1}{\epsilon_{\sigma}+2 \tilde{\delta}_{\lambda}}-\frac{U n_{\bar{\sigma}}}{\left(\epsilon_{\sigma}+2 \tilde{\delta}_{\lambda}\right)\left(\epsilon_{\sigma}+U+2 \tilde{\delta}_{\lambda}\right)}\right] \tilde{f}_{\lambda} d_{\sigma} \\
& =i \tilde{t}_{\sigma \lambda+}^{*}\left[\frac{1}{\epsilon_{\sigma}+2 \tilde{\delta}_{\lambda}}-\frac{U n_{\bar{\sigma}}}{\left(\epsilon_{\sigma}+2 \tilde{\delta}_{\lambda}\right)\left(\epsilon_{\sigma}+U+2 \tilde{\delta}_{\lambda}\right)}\right] \tilde{f}_{\lambda} d_{\sigma} .
\end{aligned}
$$

We must now calculate $\left[A_{\rho \lambda}, \tilde{H}_{T}\right]$ and $\left[B_{\rho \lambda}, \tilde{H}_{T}\right]$, involving the commutation relations

$$
\begin{aligned}
{\left[\tilde{f}_{\lambda}^{\dagger} d_{\rho}, \tilde{H}_{T}\right] } & =i \sum_{\sigma \kappa}\left[\tilde{f}_{\lambda}^{\dagger} d_{\rho}, \tilde{t}_{\sigma \kappa-}^{*} \tilde{f}_{\kappa}^{\dagger} d_{\sigma}-\tilde{t}_{\sigma \kappa+}^{*} \tilde{f}_{\kappa} d_{\sigma}-\tilde{t}_{\sigma \kappa-} d_{\sigma}^{\dagger} \tilde{f}_{\kappa}+\tilde{t}_{\sigma \kappa+} d_{\sigma}^{\dagger} \tilde{f}_{\kappa}^{\dagger}\right] \\
& =i \sum_{\sigma \kappa} \tilde{\delta}_{\kappa \lambda} \tilde{t}_{\sigma \kappa+}^{*} d_{\rho} d_{\sigma}-\tilde{t}_{\sigma \kappa-}\left(\tilde{\delta}_{\rho \sigma} \tilde{f}_{\lambda}^{\dagger} \tilde{f}_{\kappa}-\tilde{\delta}_{\lambda \kappa} d_{\sigma}^{\dagger} d_{\rho}\right)+\tilde{t}_{\sigma \kappa+} \tilde{\delta}_{\rho \sigma} \tilde{f}_{\lambda}^{\dagger} \tilde{f}_{\kappa}^{\dagger},
\end{aligned}
$$




$$
\begin{aligned}
{\left[\tilde{f}_{\lambda} d_{\rho}, \tilde{H}_{T}\right] } & =i \sum_{\sigma \kappa}\left[\tilde{f}_{\lambda} d_{\rho}, \tilde{t}_{\sigma \kappa-}^{*} \tilde{f}_{\kappa}^{\dagger} d_{\sigma}-\tilde{t}_{\sigma \kappa+}^{*} \tilde{f}_{\kappa} d_{\sigma}-\tilde{t}_{\sigma \kappa}-d_{\sigma}^{\dagger} \tilde{f}_{\kappa}+\tilde{t}_{\sigma \kappa+} d_{\sigma}^{\dagger} \tilde{f}_{\kappa}^{\dagger}\right] \\
& =i \sum_{\sigma \kappa}-\tilde{t}_{\sigma \kappa}^{*} \tilde{\delta}_{\kappa \lambda} d_{\rho} d_{\sigma}-\tilde{t}_{\sigma \kappa} \tilde{\delta}_{\rho \sigma} \tilde{f}_{\lambda} \tilde{f}_{\kappa}+\tilde{t}_{\sigma \kappa+}\left(\tilde{\delta}_{\rho \sigma} \tilde{f}_{\lambda} \tilde{f}_{\kappa}^{\dagger}-\tilde{\delta}_{\kappa \lambda} d_{\sigma}^{\dagger} d_{\rho}\right) .
\end{aligned}
$$

Note that $\left[U n_{\bar{\rho}} \tilde{f}_{\lambda}^{\dagger} d_{\rho}, \tilde{H}_{T}\right]=U n_{\bar{\rho}}\left[\tilde{f}_{\lambda}^{\dagger} d_{\rho}, \tilde{H}_{T}\right]+\left[U n_{\bar{\rho}}, \tilde{H}_{T}\right] \tilde{f}_{\lambda}^{\dagger} d_{\rho}$ and

$$
\begin{aligned}
{\left[n_{\bar{\rho}}, \tilde{H}_{T}\right] } & =i \sum_{\sigma \lambda}\left[n_{\bar{\rho}}, \tilde{t}_{\sigma \lambda-}^{*} \tilde{f}_{\lambda}^{\dagger} d_{\sigma}-\tilde{t}_{\sigma \lambda+}^{*} \tilde{f}_{\lambda} d_{\sigma}-\tilde{t}_{\sigma \lambda-} d_{\sigma}^{\dagger} \tilde{f}_{\lambda}+\tilde{t}_{\sigma \lambda+} d_{\sigma}^{\dagger} \tilde{f}_{\lambda}^{\dagger}\right] \\
& =i \sum_{\sigma \lambda} \tilde{t}_{\sigma \lambda-}^{*} \tilde{\delta}_{\bar{\rho} \sigma} d_{\bar{\rho}} \tilde{f}_{\lambda}^{\dagger}-\tilde{t}_{\sigma \lambda+}^{*} \tilde{\delta}_{\bar{\rho} \sigma} d_{\bar{\rho}} \tilde{f}_{\lambda}-\tilde{t}_{\sigma \lambda-} \tilde{\delta}_{\bar{\rho} \sigma} d_{\sigma}^{\dagger} \tilde{f}_{\lambda}+\tilde{t}_{\sigma \lambda+} \tilde{\delta}_{\bar{\rho} \sigma} d_{\sigma}^{\dagger} \tilde{f}_{\lambda}^{\dagger} .
\end{aligned}
$$

Taking the large on-site charging limit, $U \rightarrow \infty$, we find

$$
\begin{aligned}
\sum_{\rho \lambda}\left[A_{\rho \lambda}, \tilde{H}_{T}\right]= & -i \sum_{\rho \lambda} \tilde{t}_{\rho \lambda-}^{*}\left[\left(\frac{1}{\epsilon_{\rho}-2 \tilde{\delta}_{\lambda}}-\frac{n_{\bar{\rho}}}{\epsilon_{\rho}-2 \tilde{\delta}_{\lambda}}\right)\left[\tilde{f}_{\lambda}^{\dagger} d_{\rho}, \tilde{H}_{T}\right]-\frac{\left[n_{\bar{\rho}}, \tilde{H}_{T}\right] \tilde{f}_{\lambda}^{\dagger} d_{\rho}}{\epsilon_{\rho}-2 \tilde{\delta}_{\lambda}}\right] \\
= & -i \sum_{\rho \lambda} \frac{\tilde{t}_{\rho \lambda-}^{*}}{\epsilon_{\rho}-2 \tilde{\delta}_{\lambda}}\left[n_{\rho}\left[\tilde{f}_{\lambda}^{\dagger} d_{\rho}, \tilde{H}_{T}\right]-\left[n_{\bar{\rho}}, \tilde{H}_{T}\right] \tilde{f}_{\lambda}^{\dagger} d_{\rho}\right] \\
= & \sum_{\sigma \rho \kappa \lambda} \frac{\tilde{t}_{\rho \lambda-}^{*}}{\epsilon_{\rho}-2 \tilde{\delta}_{\lambda}}\left[n_{\rho}\left(\tilde{t}_{\sigma \kappa+}^{*} \tilde{\delta}_{\kappa \lambda} d_{\rho} d_{\sigma}-\tilde{t}_{\sigma \kappa-}\left(\tilde{\delta}_{\rho \sigma} \tilde{f}_{\lambda}^{\dagger} \tilde{f}_{\kappa}-\tilde{\delta}_{\kappa \lambda} d_{\sigma}^{\dagger} d_{\rho}\right)+\tilde{t}_{\sigma \kappa+} \tilde{\delta}_{\rho \sigma} \tilde{f}_{\lambda}^{\dagger} \tilde{f}_{\kappa}^{\dagger}\right)\right. \\
& \left.-\left(\tilde{t}_{\sigma \kappa-}^{*} \tilde{\delta}_{\bar{\rho} \sigma} d_{\bar{\rho}} \tilde{f}_{\kappa}^{\dagger}-\tilde{t}_{\sigma \kappa+}^{*} \tilde{\delta}_{\bar{\rho} \sigma} d_{\bar{\rho}} \tilde{f}_{\kappa}-\tilde{t}_{\sigma \kappa-} \tilde{\delta}_{\bar{\rho} \sigma} d_{\sigma}^{\dagger} \tilde{f}_{\kappa}+\tilde{t}_{\sigma \kappa+} \tilde{\delta}_{\bar{\rho} \sigma} d_{\sigma}^{\dagger} \tilde{f}_{\kappa}^{\dagger}\right) \tilde{f}_{\lambda}^{\dagger} d_{\rho}\right] \\
\sum_{\rho \lambda}\left[B_{\rho \lambda}, \tilde{H}_{T}\right]= & i \sum_{\rho \lambda} \tilde{t}_{\rho \lambda+}^{*}\left[\left(\frac{1}{\epsilon_{\rho}+2 \tilde{\delta}_{\lambda}}-\frac{n_{\bar{\rho}}}{\epsilon_{\rho}+2 \tilde{\delta}_{\lambda}}\right)\left[\tilde{f}_{\lambda} d_{\rho}, \tilde{H}_{T}\right]-\frac{\left[n_{\bar{\rho}}, \tilde{H}_{T}\right] \tilde{f}_{\lambda} d_{\rho}}{\epsilon_{\rho}+2 \tilde{\delta}_{\lambda}}\right] \\
= & i \sum_{\rho \lambda} \frac{\tilde{t}_{\rho \lambda+}^{*}}{\epsilon_{\rho}+2 \tilde{\delta}_{\lambda}}\left[n_{\rho}\left[\tilde{f}_{\lambda} d_{\rho}, \tilde{H}_{T}\right]-\left[n_{\bar{\rho}}, \tilde{H}_{T}\right] \tilde{f}_{\lambda} d_{\rho}\right] \\
= & -\sum_{\sigma \rho \kappa \lambda} \frac{\tilde{t}_{\rho \lambda+}^{*}}{\epsilon_{\rho}+2 \tilde{\delta}_{\lambda}}\left\{n_{\rho}\left[-\tilde{t}_{\sigma \kappa}^{*} \tilde{\delta}_{\kappa \lambda} d_{\rho} d_{\sigma}-\tilde{t}_{\sigma \kappa-} \tilde{\delta}_{\rho \sigma} \tilde{f}_{\lambda} \tilde{f}_{\kappa}+\tilde{t}_{\sigma \kappa+}\left(\tilde{\delta}_{\rho \sigma} \tilde{f}_{\lambda} \tilde{f}_{\kappa}^{\dagger}-\tilde{\delta}_{\kappa \lambda} d_{\sigma}^{\dagger} d_{\rho}\right)\right]\right. \\
& \left.-\left(\tilde{t}_{\sigma \kappa-}^{*} \tilde{\delta}_{\bar{\rho} \sigma} d_{\bar{\rho}} \tilde{f}_{\kappa}^{\dagger}-\tilde{t}_{\sigma \kappa+}^{*} \tilde{\delta}_{\bar{\rho} \sigma} d_{\bar{\rho}} \tilde{f}_{\kappa}-\tilde{t}_{\sigma \kappa} \tilde{\delta}_{\bar{\rho} \sigma} d_{\sigma}^{\dagger} \tilde{f}_{\kappa}+\tilde{t}_{\sigma \kappa+} \tilde{\delta}_{\bar{\rho} \sigma} d_{\sigma}^{\dagger} \tilde{f}_{\kappa}^{\dagger}\right) \tilde{f}_{\lambda} d_{\rho}\right\} .
\end{aligned}
$$

Notice that, for $\hat{O}=\tilde{f}_{\lambda}^{\dagger}, \tilde{f}_{\lambda}, n_{\rho}\left[\hat{O} d_{\rho}, \tilde{H}_{T}\right]=-n_{\rho} \tilde{H}_{T} \hat{O} d_{\rho}$. The only term that survives from $\tilde{H}_{T}$ is proportional to $d_{\rho}^{\dagger}$ so that this term has no spin flip processes:

$$
\begin{aligned}
& -n_{\rho} \tilde{H}_{T} \tilde{f}_{\lambda}^{\dagger} d_{\rho}=i\left(\tilde{t}_{\rho \kappa}-n_{\rho} d_{\rho}^{\dagger} \tilde{f}_{\kappa}-\tilde{t}_{\rho \kappa+} d_{\rho}^{\dagger} \tilde{f}_{\kappa}^{\dagger}\right) \tilde{f}_{\lambda}^{\dagger} d_{\rho}=i\left(\tilde{t}_{\rho \kappa-} \tilde{f}_{\kappa} \tilde{f}_{\lambda}^{\dagger}-\tilde{t}_{\rho \kappa+} \tilde{f}_{\kappa}^{\dagger} \tilde{f}_{\lambda}^{\dagger}\right) n_{\rho}, \\
& -n_{\rho} \tilde{H}_{T} \tilde{f}_{\lambda} d_{\rho}=-i\left(-\tilde{t}_{\rho \kappa+} n_{\rho} d_{\rho}^{\dagger} \tilde{f}_{\kappa}^{\dagger}+\tilde{t}_{\rho \kappa} d_{\rho}^{\dagger} \tilde{f}_{\kappa}\right) \tilde{f}_{\lambda} d_{\rho}=-i\left(-\tilde{t}_{\rho \kappa+} \tilde{f}_{\kappa}^{\dagger} \tilde{f}_{\lambda}+\tilde{t}_{\rho \kappa}-\tilde{f}_{\kappa} \tilde{f}_{\lambda}\right) n_{\rho} .
\end{aligned}
$$

Therefore, these terms do not involve spin flips and

$$
\begin{aligned}
\sum_{\rho \lambda}\left[A_{\rho \lambda}, \tilde{H}_{T}\right] & =-i \sum_{\rho \lambda} \tilde{t}_{\rho \lambda-}^{*}\left[\left(\frac{1}{\epsilon_{\rho}-2 \tilde{\delta}_{\lambda}}-\frac{n_{\bar{\rho}}}{\epsilon_{\rho}-2 \tilde{\delta}_{\lambda}}\right)\left[\tilde{f}_{\lambda}^{\dagger} d_{\rho}, \tilde{H}_{T}\right]-\frac{\left[n_{\bar{\rho}}, \tilde{H}_{T}\right] \tilde{f}_{\lambda}^{\dagger} d_{\rho}}{\epsilon_{\rho}-2 \tilde{\delta}_{\lambda}}\right] \\
& =-i \sum_{\rho \lambda} \frac{\tilde{t}_{\rho \lambda-}^{*}}{\epsilon_{\rho}-2 \tilde{\delta}_{\lambda}}\left[n_{\rho}\left[\tilde{f}_{\lambda}^{\dagger} d_{\rho}, \tilde{H}_{T}\right]-\left[n_{\bar{\rho}}, \tilde{H}_{T}\right] \tilde{f}_{\lambda}^{\dagger} d_{\rho}\right] \\
& =\sum_{\sigma \rho \kappa \lambda} \frac{\tilde{t}_{\rho \lambda-}^{*}}{\epsilon_{\rho}-2 \tilde{\delta}_{\lambda}}\left[\left(\tilde{t}_{\sigma \kappa}-\tilde{f}_{\kappa} \tilde{f}_{\lambda}^{\dagger}-\tilde{t}_{\sigma \kappa+} \tilde{f}_{\kappa}^{\dagger} \tilde{f}_{\lambda}^{\dagger} \tilde{\delta}_{\sigma \rho} n_{\rho}\right.\right. \\
& \left.-\left(\tilde{t}_{\sigma \kappa-}^{*} \tilde{\delta}_{\bar{\rho} \sigma} d_{\bar{\rho}} \tilde{f}_{\kappa}^{\dagger}-\tilde{t}_{\sigma \kappa+}^{*} \tilde{\delta}_{\bar{\rho} \sigma} d_{\bar{\rho}} \tilde{f}_{\kappa}-\tilde{t}_{\sigma \kappa}-\tilde{\delta}_{\bar{\rho} \sigma} d_{\sigma}^{\dagger} \tilde{f}_{\kappa}+\tilde{t}_{\sigma \kappa+} \tilde{\delta}_{\bar{\rho} \sigma} d_{\sigma}^{\dagger} \tilde{f}_{\kappa}^{\dagger}\right) \tilde{f}_{\lambda}^{\dagger} d_{\rho}\right] \\
& =\sum_{\sigma \rho \kappa \lambda} \frac{\tilde{t}_{\rho \lambda-}^{*}}{\epsilon_{\rho}-2 \tilde{\delta}_{\lambda}}\left[\tilde{t}_{\sigma \kappa}-\tilde{\delta}_{\sigma \rho} n_{\rho} \tilde{f}_{\kappa} \tilde{f}_{\lambda}^{\dagger}-\tilde{t}_{\sigma \kappa+} \tilde{\delta}_{\sigma \rho} n_{\rho} \tilde{f}_{\kappa}^{\dagger} \tilde{f}_{\lambda}^{\dagger}-\left(-\tilde{t}_{\sigma \kappa}-\tilde{\delta}_{\bar{\rho} \sigma} d_{\sigma}^{\dagger} \tilde{f}_{\kappa}+\tilde{t}_{\sigma \kappa+} \tilde{\delta}_{\bar{\rho} \sigma} d_{\sigma}^{\dagger} \tilde{f}_{\kappa}^{\dagger}\right) \tilde{f}_{\lambda}^{\dagger} d_{\rho}\right] \\
\sum_{\rho \lambda}\left[B_{\rho \lambda}, \tilde{H}_{T}\right] & =i \sum_{\rho \lambda} \tilde{t}_{\rho \lambda+}^{*}\left[\left(\frac{1}{\epsilon_{\rho}+2 \tilde{\delta}_{\lambda}}-\frac{n_{\bar{\rho}}}{\epsilon_{\rho}+2 \tilde{\delta}_{\lambda}}\right)\left[\tilde{f}_{\lambda} d_{\rho}, \tilde{H}_{T}\right]-\frac{\left.\left[n_{\bar{\rho}}, \tilde{H}_{T}\right] \tilde{f}_{\lambda} d_{\rho}\right]}{\epsilon_{\rho}+2 \tilde{\delta}_{\lambda}}\right]
\end{aligned}
$$




$$
\begin{aligned}
= & i \sum_{\rho \lambda} \frac{\tilde{t}_{\rho \lambda+}^{*}}{\epsilon_{\rho}+2 \tilde{\delta}_{\lambda}}\left[n_{\rho}\left[\tilde{f}_{\lambda} d_{\rho}, \tilde{H}_{T}\right]-\left[n_{\bar{\rho}}, \tilde{H}_{T}\right] \tilde{f}_{\lambda} d_{\rho}\right] \\
= & -\sum_{\sigma \rho \kappa \lambda} \frac{\tilde{t}_{\rho \lambda+}^{*}}{\epsilon_{\rho}+2 \tilde{\delta}_{\lambda}}\left[-\tilde{t}_{\sigma \kappa}+\tilde{\delta}_{\sigma \rho} n_{\rho} \tilde{f}_{\kappa}^{\dagger} \tilde{f}_{\lambda}+\tilde{t}_{\sigma \kappa} \tilde{\delta}_{\sigma \rho} n_{\rho} \tilde{f}_{\kappa} \tilde{f}_{\lambda}\right. \\
& \left.-\left(\tilde{t}_{\sigma \kappa-}^{*} \tilde{\delta}_{\bar{\rho} \sigma} d_{\bar{\rho}} \tilde{f}_{\kappa}^{\dagger}-\tilde{t}_{\sigma \kappa+}^{*} \tilde{\delta}_{\bar{\rho} \sigma} d_{\bar{\rho}} \tilde{f}_{\kappa}-\tilde{t}_{\sigma \kappa} \tilde{\delta}_{\bar{\rho} \sigma} d_{\sigma}^{\dagger} \tilde{f}_{\kappa}+\tilde{t}_{\sigma \kappa+} \tilde{\delta}_{\bar{\rho} \sigma} d_{\sigma}^{\dagger} \tilde{f}_{\kappa}^{\dagger}\right) \tilde{f}_{\lambda} d_{\rho}\right] \\
= & -\sum_{\sigma \rho \kappa \lambda} \frac{\tilde{t}_{\rho \lambda+}^{*}}{\epsilon_{\rho}+2 \tilde{\delta}_{\lambda}}\left[-\tilde{t}_{\sigma \kappa+} \tilde{\delta}_{\sigma \rho} n_{\rho} \tilde{f}_{\kappa}^{\dagger} \tilde{f}_{\lambda}+\tilde{t}_{\sigma \kappa} \tilde{\delta}_{\sigma \rho} n_{\rho} \tilde{f}_{\kappa} \tilde{f}_{\lambda}-\left(-\tilde{t}_{\sigma \kappa}-\tilde{\delta}_{\bar{\rho} \sigma} d_{\sigma}^{\dagger} \tilde{f}_{\kappa}+\tilde{t}_{\sigma \kappa+} \tilde{\delta}_{\bar{\rho} \sigma} d_{\sigma}^{\dagger} \tilde{f}_{\kappa}^{\dagger}\right) \tilde{f}_{\lambda} d_{\rho}\right] .
\end{aligned}
$$

Let us consider processes when only one wire is involved in then tunneling, $\kappa=\lambda$ :

$$
\begin{aligned}
\sum_{\rho \lambda}\left[A_{\rho \lambda}, \tilde{H}_{T}\right] & =\sum_{\sigma \rho \kappa \lambda} \frac{\tilde{t}_{\rho \lambda-}^{*}}{\epsilon_{\rho}-2 \tilde{\delta}_{\lambda}}\left[\tilde{t}_{\sigma \kappa}-\tilde{\delta}_{\sigma \rho} n_{\rho} \tilde{f}_{\kappa} \tilde{f}_{\lambda}^{\dagger}-\tilde{t}_{\sigma \kappa+} \tilde{\delta}_{\sigma \rho} n_{\rho} \tilde{f}_{\kappa}^{\dagger} \tilde{f}_{\lambda}^{\dagger}-\left(-\tilde{t}_{\sigma \kappa}-\tilde{\delta}_{\bar{\rho} \sigma} d_{\sigma}^{\dagger} \tilde{f}_{\kappa}+\tilde{t}_{\sigma \kappa+} \tilde{\delta}_{\bar{\rho} \sigma} d_{\sigma}^{\dagger} \tilde{f}_{\kappa}^{\dagger}\right) \tilde{f}_{\lambda}^{\dagger} d_{\rho}\right] \\
& =\sum_{\rho \lambda} \frac{\tilde{t}_{\rho \lambda-}^{*}}{\epsilon_{\rho}-2 \tilde{\delta}_{\lambda}}\left[\tilde{t}_{\rho \lambda-} n_{\rho} \tilde{f}_{\lambda} \tilde{f}_{\lambda}^{\dagger}+\tilde{t}_{\bar{\rho} \lambda-} d_{\bar{\rho}}^{\dagger} \tilde{f}_{\lambda} \tilde{f}_{\lambda}^{\dagger} d_{\rho}\right] \\
\sum_{\rho \lambda}\left[B_{\rho \lambda}, \tilde{H}_{T}\right] & =-\sum_{\sigma \rho \kappa \lambda} \frac{\tilde{t}_{\rho \lambda+}^{*}}{\epsilon_{\rho}+2 \tilde{\delta}_{\lambda}}\left[-\tilde{t}_{\sigma \kappa}+\tilde{\delta}_{\sigma \rho} n_{\rho} \tilde{f}_{\kappa}^{\dagger} \tilde{f}_{\lambda}+\tilde{t}_{\sigma \kappa-} \tilde{\delta}_{\sigma \rho} n_{\rho} \tilde{f}_{\kappa} \tilde{f}_{\lambda}-\left(-\tilde{t}_{\sigma \kappa-} \tilde{\delta}_{\bar{\rho} \sigma} d_{\sigma}^{\dagger} \tilde{f}_{\kappa}+\tilde{t}_{\sigma \kappa+} \tilde{\delta}_{\bar{\rho} \sigma} d_{\sigma}^{\dagger} \tilde{f}_{\kappa}^{\dagger}\right) \tilde{f}_{\lambda} d_{\rho}\right] \\
& =-\sum_{\rho \lambda} \frac{\tilde{t}_{\rho \lambda+}^{*}}{\epsilon_{\rho}+2 \tilde{\delta}}\left[-\tilde{t}_{\rho \lambda+} n_{\rho} \tilde{f}_{\lambda}^{\dagger} \tilde{f}_{\lambda}-\tilde{t}_{\bar{\rho} \lambda+} \tilde{f}_{\lambda} d_{\bar{\rho}}^{\dagger} \tilde{f}_{\lambda}^{\dagger} \tilde{f}_{\lambda} d_{\rho}\right] \\
& =\sum_{\rho \lambda} \frac{\tilde{t}_{\rho \lambda+}^{*}}{\epsilon_{\rho}+2 \tilde{\delta}}\left[\tilde{t}_{\rho \lambda+} n_{\rho} \tilde{f}_{\lambda}^{\dagger} \tilde{f}_{\lambda}+\tilde{t}_{\bar{\rho} \lambda+} d_{\bar{\rho}}^{\dagger} \tilde{f}_{\lambda}^{\dagger} \tilde{f}_{\lambda} d_{\rho}\right] .
\end{aligned}
$$

Summing these together with their Hermitian conjugate, we get

$$
\begin{aligned}
\tilde{\mathcal{H}}_{s}= & \sum_{\rho \lambda} 2 n_{\rho}\left(\frac{\left|\tilde{t}_{\rho \lambda+}\right|^{2}}{\epsilon_{\rho}+2 \tilde{\delta}_{\lambda}} \tilde{f}_{\lambda}^{\dagger} \tilde{f}_{\lambda}+\frac{\left|\tilde{t}_{\rho \lambda-}\right|^{2}}{\epsilon_{\rho}-2 \tilde{\delta}_{\lambda}} \tilde{f}_{\lambda} \tilde{f}_{\lambda}^{\dagger}\right)+d_{\bar{\rho}}^{\dagger} d_{\rho}\left(\frac{\tilde{t}_{\rho \lambda+}^{*} \tilde{t}_{\bar{\rho} \lambda+t}}{\epsilon_{\rho}+2 \tilde{\delta}_{\lambda}} \tilde{f}_{\lambda}^{\dagger} \tilde{f}_{\lambda}+\frac{\tilde{t}_{\rho \lambda-}^{*} \tilde{t}_{\bar{\rho} \lambda-}}{\epsilon_{\rho}-2 \tilde{\delta}_{\lambda}} \tilde{f}_{\lambda} \tilde{f}_{\lambda}^{\dagger}\right) \\
& +d_{\bar{\rho}}^{\dagger} d_{\rho}\left(\frac{\tilde{t}_{\rho \lambda+}^{*} \tilde{t}_{\bar{\rho} \lambda+}}{\epsilon_{\bar{\rho}}+2 \tilde{\delta}_{\lambda}} \tilde{f}_{\lambda}^{\dagger} \tilde{f}_{\lambda}+\frac{\tilde{t}_{\rho \lambda-}^{*} \tilde{t}_{\bar{\rho} \lambda-}}{\epsilon_{\bar{\rho}}-2 \tilde{\delta}_{\lambda}} \tilde{f}_{\lambda} \tilde{f}_{\lambda}^{\dagger}\right) \\
= & \sum_{\rho \lambda} 2 n_{\rho}\left(\frac{\left|\tilde{t}_{\rho \lambda+}\right|^{2}}{\epsilon_{\rho}+2 \tilde{\delta}_{\lambda}} \tilde{f}_{\lambda}^{\dagger} \tilde{f}_{\lambda}+\frac{\left|\tilde{t}_{\rho \lambda-}\right|^{2}}{\epsilon_{\rho}-2 \tilde{\delta}_{\lambda}} \tilde{f}_{\lambda} \tilde{f}_{\lambda}^{\dagger}\right)+d_{\rho}^{\dagger} d_{\bar{\rho}}\left(\frac{\tilde{t}_{\bar{\rho} \lambda+}^{*} \tilde{f}_{\rho \lambda+}}{\epsilon_{\bar{\rho}}+2 \tilde{\delta}_{\lambda}} \tilde{f}_{\lambda}^{\dagger} \tilde{f}_{\lambda}+\frac{\tilde{t}_{\bar{\rho} \lambda-}^{*} \tilde{t}_{\rho \lambda-}}{\epsilon_{\bar{\rho}}-2 \tilde{\delta}_{\lambda}} \tilde{f}_{\lambda} \tilde{f}_{\lambda}^{\dagger}\right) \\
& +d_{\rho}^{\dagger} d_{\bar{\rho}}\left(\frac{\tilde{t}_{\bar{\rho} \lambda+}^{*} \tilde{t}_{\rho \lambda+}}{\epsilon_{\rho}+2 \tilde{\delta}_{\lambda}} \tilde{f}_{\lambda}^{\dagger} \tilde{f}_{\lambda}+\frac{\tilde{t}_{\bar{\rho} \lambda-}^{*} \tilde{t}_{\rho \lambda-}}{\epsilon_{\rho}-2 \tilde{\delta}_{\lambda}} \tilde{f_{\lambda}} \tilde{f}_{\lambda}^{\dagger}\right) \\
= & \sum_{\rho \lambda}\left[2 n_{\rho}\left(\frac{\left|\tilde{t}_{\rho \lambda+}\right|^{2}}{\epsilon_{\rho}+2 \tilde{\delta}_{\lambda}}-\frac{\left|\tilde{t}_{\rho \lambda-}\right|^{2}}{\epsilon_{\rho}-2 \tilde{\delta}_{\lambda}}\right)+d_{\rho}^{\dagger} d_{\bar{\rho}}\left(\frac{\tilde{t}_{\bar{\rho} \lambda+}^{*} \tilde{t}_{\rho \lambda+}}{\epsilon_{\bar{\rho}}+2 \tilde{\delta}_{\lambda}}+\frac{\tilde{t}_{\bar{\rho} \lambda+}^{*} \tilde{t}_{\rho \lambda+}}{\epsilon_{\rho}+2 \tilde{\delta}_{\lambda}}-\frac{\tilde{t}_{\bar{\rho} \lambda-}^{*} \tilde{t}_{\rho \lambda-}}{\epsilon_{\bar{\rho}}-2 \tilde{\delta}_{\lambda}}-\frac{\tilde{t}_{\bar{\rho} \lambda-}^{*} \tilde{t}_{\rho \lambda-}}{\epsilon_{\rho}-2 \tilde{\delta}_{\lambda}}\right)\right] \tilde{f}_{\lambda}^{\dagger} \tilde{f}_{\lambda} \\
& +2 n_{\rho} \frac{\left|\tilde{t}_{\rho \lambda-}\right|^{2}}{\epsilon_{\rho}-2 \tilde{\delta}_{\lambda}}+d_{\rho}^{\dagger} d_{\bar{\rho}}\left(\frac{\tilde{t}_{\bar{\rho} \lambda-}^{*} \tilde{t}_{\rho \lambda-}}{\epsilon_{\bar{\rho}}-2 \tilde{\delta}_{\lambda}}+\frac{\tilde{t}_{\bar{\rho} \lambda-}^{*} \tilde{t}_{\rho \lambda-}}{\epsilon_{\rho}-2 \tilde{\delta}_{\lambda}}\right) .
\end{aligned}
$$

Processes involving two wires, $\kappa=\bar{\lambda}$, are calculated from

$$
\begin{aligned}
\sum_{\rho \lambda}\left[A_{\rho \lambda}, \tilde{H}_{T}\right] & =\sum_{\sigma \rho \kappa \lambda} \frac{\tilde{t}_{\rho \lambda-}^{*}}{\epsilon_{\rho}-2 \tilde{\delta}_{\lambda}}\left[\tilde{t}_{\sigma \kappa}-\tilde{\delta}_{\sigma \rho} n_{\rho} \tilde{f}_{\kappa} \tilde{f}_{\lambda}^{\dagger}-\tilde{t}_{\sigma \kappa+} \tilde{\delta}_{\sigma \rho} n_{\rho} \tilde{f}_{\kappa}^{\dagger} \tilde{f}_{\lambda}^{\dagger}-\left(-\tilde{t}_{\sigma \kappa}-\tilde{\delta}_{\bar{\rho} \sigma} d_{\sigma}^{\dagger} \tilde{f}_{\kappa}+\tilde{t}_{\sigma \kappa}+\tilde{\delta}_{\bar{\rho} \sigma} d_{\sigma}^{\dagger} \tilde{f}_{\kappa}^{\dagger}\right) \tilde{f}_{\lambda}^{\dagger} d_{\rho}\right] \\
& =\sum_{\rho \lambda} \frac{\tilde{t}_{\rho \lambda-}^{*}}{\epsilon_{\rho}-2 \tilde{\delta}_{\lambda}}\left[\tilde{t}_{\rho \bar{\lambda}} n_{\rho} \tilde{f}_{\bar{\lambda}} \tilde{f}_{\lambda}^{\dagger}-\tilde{t}_{\rho \bar{\lambda}+} n_{\rho} \tilde{f}_{\bar{\lambda}}^{\dagger} \tilde{f}_{\lambda}^{\dagger}-\left(-\tilde{t}_{\bar{\rho} \bar{\lambda}-} d_{\bar{\rho}}^{\dagger} \tilde{f}_{\bar{\lambda}}+\tilde{t}_{\bar{\rho} \bar{\lambda}+} d_{\bar{\rho}}^{\dagger} \tilde{f}_{\bar{\lambda}}^{\dagger}\right) \tilde{f}_{\lambda}^{\dagger} d_{\rho}\right] \\
\sum_{\rho \lambda}\left[B_{\rho \lambda}, \tilde{H}_{T}\right] & =-\sum_{\sigma \rho \kappa \lambda} \frac{\tilde{t}_{\rho \lambda+}^{*}}{\epsilon_{\rho}+2 \tilde{\delta}_{\lambda}}\left[-\tilde{t}_{\sigma \kappa+} \tilde{\delta}_{\sigma \rho} n_{\rho} \tilde{f}_{\kappa}^{\dagger} \tilde{f}_{\lambda}+\tilde{t}_{\sigma \kappa} \tilde{\delta}_{\sigma \rho} n_{\rho} \tilde{f}_{\kappa} \tilde{f}_{\lambda}-\left(-\tilde{t}_{\sigma \kappa}-\tilde{\delta}_{\bar{\rho} \sigma} d_{\sigma}^{\dagger} \tilde{f}_{\kappa}+\tilde{t}_{\sigma \kappa}+\tilde{\delta}_{\bar{\rho} \sigma} d_{\sigma}^{\dagger} \tilde{f}_{\kappa}^{\dagger}\right) \tilde{f}_{\lambda} d_{\rho}\right]
\end{aligned}
$$




$$
\begin{aligned}
& =-\sum_{\rho \lambda} \frac{\tilde{t}_{\rho \lambda+}^{*}}{\epsilon_{\rho}+2 \tilde{\delta}_{\lambda}}\left[-\tilde{t}_{\rho \bar{\lambda}+} n_{\rho} \tilde{f}_{\bar{\lambda}}^{\dagger} \tilde{f}_{\lambda}+\tilde{t}_{\rho \bar{\lambda}} n_{\rho} \tilde{f}_{\bar{\lambda}} \tilde{f}_{\bar{\lambda}}-\left(-\tilde{t}_{\bar{\rho} \bar{\lambda}-} d_{\bar{\rho}}^{\dagger} \tilde{f}_{\bar{\lambda}}+\tilde{t}_{\bar{\rho} \bar{\lambda}+} d_{\bar{\rho}}^{\dagger} \tilde{f}_{\bar{\lambda}}^{\dagger}\right) \tilde{f}_{\lambda} d_{\rho}\right]
\end{aligned}
$$

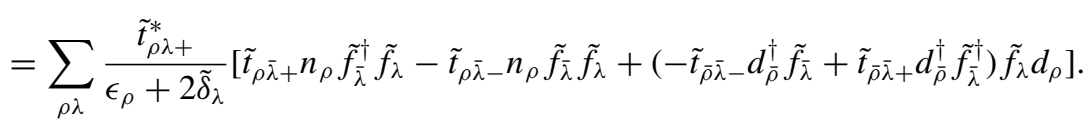

Because we will have to add the Hermitian conjugates of these terms, we note that

$$
\begin{aligned}
& {\left[\sum_{\rho \lambda} \frac{\tilde{t}_{\rho \lambda-}^{*}}{\epsilon_{\rho}-2 \tilde{\delta}_{\lambda}} \tilde{t}_{\rho \bar{\lambda}-} n_{\rho} \tilde{f}_{\bar{\lambda}} \tilde{f}_{\lambda}^{\dagger}\right]^{\dagger}=\sum_{\rho \lambda} \frac{\tilde{t}_{\rho \lambda-}^{*}}{\epsilon_{\rho}-2 \tilde{\delta}_{\bar{\lambda}}} \tilde{t}_{\rho \bar{\lambda}-} n_{\rho} \tilde{f}_{\bar{\lambda}} \tilde{f}_{\lambda}^{\dagger},} \\
& {\left[\sum_{\rho \lambda} \frac{\tilde{t}_{\rho \lambda+}^{*}}{\epsilon_{\rho}+2 \tilde{\delta}_{\lambda}} \tilde{t}_{\rho \bar{\lambda}+} n_{\rho} \tilde{f}_{\bar{\lambda}}^{\dagger} \tilde{f}_{\lambda}\right]^{\dagger}=\sum_{\rho \lambda} \frac{\tilde{t}_{\rho \lambda+}^{*}}{\epsilon_{\rho}+2 \tilde{\delta}_{\bar{\lambda}}} \tilde{t}_{\rho \bar{\lambda}+} n_{\rho} \tilde{f}_{\bar{\lambda}}^{\dagger} \tilde{f}_{\lambda},} \\
& {\left[\sum_{\rho \lambda} \frac{\tilde{t}_{\rho \lambda-}^{*}}{\epsilon_{\rho}-2 \tilde{\delta}_{\lambda}} \tilde{t}_{\bar{\rho} \bar{\lambda}-} d_{\bar{\rho}}^{\dagger} d_{\rho} \tilde{f}_{\bar{\lambda}} \tilde{f}_{\lambda}^{\dagger}\right]^{\dagger}=\sum_{\rho \lambda} \frac{\tilde{t}_{\rho \lambda-}^{*}}{\epsilon_{\bar{\rho}}-2 \tilde{\delta}_{\bar{\lambda}}} \tilde{t}_{\bar{\rho} \bar{\lambda}-} d_{\bar{\rho}}^{\dagger} d_{\rho} \tilde{f}_{\bar{\lambda}} \tilde{f}_{\lambda}^{\dagger},} \\
& {\left[\sum_{\rho \lambda} \frac{\tilde{t}_{\rho \lambda+}^{*}}{\epsilon_{\rho}+2 \tilde{\delta}_{\lambda}} \tilde{t}_{\bar{\rho} \bar{\lambda}+} d_{\bar{\rho}}^{\dagger} d_{\rho} \tilde{f}_{\bar{\lambda}}^{\dagger} \tilde{f}_{\lambda}\right]^{\dagger}=\sum_{\rho \lambda} \frac{\tilde{t}_{\rho \lambda+}^{*}}{\epsilon_{\bar{\rho}}+2 \tilde{\delta}_{\bar{\lambda}}} \tilde{t}_{\bar{\rho} \bar{\lambda}+} d_{\bar{\rho}}^{\dagger} d_{\rho} \tilde{f}_{\bar{\lambda}}^{\dagger} \tilde{f}_{\lambda},}
\end{aligned}
$$

so that the contribution from the transfer of the fermions [Figs. 2(c) and 2(d)] is

$$
\begin{aligned}
& \tilde{\mathcal{H}}_{o}=\sum_{\rho \lambda}\left[\left(\frac{1}{\epsilon_{\rho}-2 \tilde{\delta}_{\bar{\lambda}}}+\frac{1}{\epsilon_{\rho}-2 \tilde{\delta}_{\lambda}}\right) \tilde{t}_{\rho \lambda-} \tilde{t}_{\rho \bar{\lambda}-}^{*} \tilde{f}_{\lambda} \tilde{f}_{\bar{\lambda}}^{\dagger}+\left(\frac{1}{\epsilon_{\rho}+2 \tilde{\delta}_{\bar{\lambda}}}+\frac{1}{\epsilon_{\rho}+2 \tilde{\delta}_{\lambda}}\right) \tilde{t}_{\rho \lambda+} \tilde{t}_{\rho \bar{\lambda}+}^{*} \tilde{f}_{\lambda}^{\dagger} \tilde{f}_{\bar{\lambda}}\right] n_{\rho} \\
& +\left[\left(\frac{1}{\epsilon_{\rho}-2 \tilde{\delta}_{\bar{\lambda}}}+\frac{1}{\epsilon_{\bar{\rho}}-2 \tilde{\delta}_{\lambda}}\right) \tilde{t}_{\rho \bar{\lambda}-}^{*} \tilde{t}_{\bar{\rho} \lambda-} \tilde{f}_{\lambda} \tilde{f}_{\bar{\lambda}}^{\dagger}+\left(\frac{1}{\epsilon_{\rho}+2 \tilde{\delta}_{\bar{\lambda}}}+\frac{1}{\epsilon_{\bar{\rho}}+2 \tilde{\delta}_{\lambda}}\right) \tilde{t}_{\rho \bar{\lambda}+}^{*} \tilde{t}_{\bar{\rho} \lambda+} \tilde{f}_{\lambda}^{\dagger} \tilde{f}_{\bar{\lambda}}\right] d_{\bar{\rho}}^{\dagger} d_{\rho} \\
& =\sum_{\rho \lambda}\left[\left(\frac{1}{\epsilon_{\rho}-2 \tilde{\delta}_{\lambda}}+\frac{1}{\epsilon_{\rho}-2 \tilde{\delta}_{\bar{\lambda}}}\right) \tilde{t}_{\rho \bar{\lambda}-} \tilde{t}_{\rho \lambda-}^{*} \tilde{f}_{\bar{\lambda}} \tilde{f}_{\lambda}^{\dagger}+\left(\frac{1}{\epsilon_{\rho}+2 \tilde{\delta}_{\bar{\lambda}}}+\frac{1}{\epsilon_{\rho}+2 \tilde{\delta}_{\lambda}}\right) \tilde{t}_{\rho \lambda+} \tilde{t}_{\rho \bar{\lambda}+}^{*} \tilde{f}_{\lambda}^{\dagger} \tilde{f}_{\bar{\lambda}}\right] n_{\rho} \\
& +\left[\left(\frac{1}{\epsilon_{\rho}-2 \tilde{\delta}_{\lambda}}+\frac{1}{\epsilon_{\bar{\rho}}-2 \tilde{\delta}_{\bar{\lambda}}}\right) \tilde{t}_{\rho \lambda-}^{*} \tilde{t}_{\bar{\rho} \bar{\lambda}-} \tilde{f}_{\bar{\lambda}} \tilde{f}_{\lambda}^{\dagger}+\left(\frac{1}{\epsilon_{\rho}+2 \tilde{\delta}_{\bar{\lambda}}}+\frac{1}{\epsilon_{\bar{\rho}}+2 \tilde{\delta}_{\lambda}}\right) \tilde{t}_{\rho \bar{\lambda}+}^{*} \tilde{t}_{\bar{\rho} \lambda+} \tilde{f}_{\lambda}^{\dagger} \tilde{f}_{\bar{\lambda}}\right] d_{\bar{\rho}}^{\dagger} d_{\rho} \\
& =\sum_{\rho \lambda}\left\{\left[\left(\frac{1}{\epsilon_{\rho}+2 \tilde{\delta}_{\bar{\lambda}}}+\frac{1}{\epsilon_{\rho}+2 \tilde{\delta}_{\lambda}}\right) \tilde{t}_{\rho \lambda+} \tilde{t}_{\rho \bar{\lambda}+}^{*}-\left(\frac{1}{\epsilon_{\rho}-2 \tilde{\delta}_{\lambda}}+\frac{1}{\epsilon_{\rho}-2 \tilde{\delta}_{\bar{\lambda}}}\right) \tilde{t}_{\rho \bar{\lambda}-} \tilde{t}_{\rho \lambda-}^{*}\right] n_{\rho}\right. \\
& \left.+\left[\left(\frac{1}{\epsilon_{\rho}+2 \tilde{\delta}_{\bar{\lambda}}}+\frac{1}{\epsilon_{\bar{\rho}}+2 \tilde{\delta}_{\lambda}}\right) \tilde{t}_{\rho \bar{\lambda}+}^{*} \tilde{t}_{\bar{\rho} \lambda+}-\left(\frac{1}{\epsilon_{\rho}-2 \tilde{\delta}_{\lambda}}+\frac{1}{\epsilon_{\bar{\rho}}-2 \tilde{\delta}_{\bar{\lambda}}}\right) \tilde{t}_{\rho \lambda-}^{*} \tilde{t}_{\bar{\rho} \bar{\lambda}-}\right] d_{\bar{\rho}}^{\dagger} d_{\rho}\right\} \tilde{f}_{\lambda}^{\dagger} \tilde{\bar{\tau}}_{\bar{\lambda}} \\
& =\sum_{\rho \lambda}\left\{\left[\left(\frac{1}{\epsilon_{\rho}+2 \tilde{\delta}_{\bar{\lambda}}}+\frac{1}{\epsilon_{\rho}+2 \tilde{\delta}_{\lambda}}\right) \tilde{t}_{\rho \lambda+} \tilde{t}_{\rho \bar{\lambda}+}^{*}-\left(\frac{1}{\epsilon_{\rho}-2 \tilde{\delta}_{\lambda}}+\frac{1}{\epsilon_{\rho}-2 \tilde{\delta}_{\bar{\lambda}}}\right) \tilde{t}_{\rho \bar{\lambda}-} \tilde{t}_{\rho \lambda-}^{*}\right] n_{\rho}\right. \\
& \left.+\left[\left(\frac{1}{\epsilon_{\bar{\rho}}+2 \tilde{\delta}_{\bar{\lambda}}}+\frac{1}{\epsilon_{\rho}+2 \tilde{\delta}_{\lambda}}\right) \tilde{t}_{\bar{\rho} \bar{\lambda}+}^{*} \tilde{t}_{\rho \lambda+}-\left(\frac{1}{\epsilon_{\bar{\rho}}-2 \tilde{\delta}_{\lambda}}+\frac{1}{\epsilon_{\rho}-2 \tilde{\delta}_{\bar{\lambda}}}\right) \tilde{t}_{\bar{\rho} \lambda-}^{*} \tilde{t}_{\rho \bar{\lambda}-}\right] d_{\rho}^{\dagger} d_{\bar{\rho}}\right\} \tilde{f}_{\lambda}^{\dagger} \tilde{f}_{\bar{\lambda}} .
\end{aligned}
$$

Instead of forming Dirac fermions in the same wire, one can instead form a full fermion from the MBSs closest together (inner fermion) and a fermion from the MBSs furthest apart (outer fermion) as in the main text $f_{r}=\left(\gamma_{r}^{\prime}+i \gamma_{l}\right) / 2$ and $f_{l}=\left(\gamma_{l}^{\prime}+i \gamma_{r}\right) / 2$, respectively. The MBSs are, in turn, written as $\gamma_{\lambda}^{\prime}=f_{\lambda}+f_{\lambda}^{\dagger}$ and $\gamma_{\lambda}=\left(f_{\bar{\lambda}}-f_{\bar{\lambda}}^{\dagger}\right) / i$.

The tunneling Hamiltonian can then be written as

$$
\begin{aligned}
H_{T} & =\sum_{\sigma, \lambda} i t_{\sigma \lambda}^{\prime} d_{\sigma}^{\dagger}\left(f_{\lambda}+f_{\lambda}^{\dagger}\right)-i t_{\sigma \lambda} d_{\sigma}^{\dagger}\left(f_{\bar{\lambda}}-f_{\bar{\lambda}}^{\dagger}\right)-i t_{\sigma \lambda}^{*}\left(f_{\bar{\lambda}}-f_{\bar{\lambda}}^{\dagger}\right) d_{\sigma}-i t_{\sigma \lambda}^{\prime}\left(f_{\lambda}+f_{\lambda}^{\dagger}\right) d_{\sigma} \\
& =\sum_{\sigma, \lambda} i d_{\sigma}^{\dagger}\left[\left(t_{\sigma \lambda}^{\prime}-t_{\bar{\lambda}}\right) f_{\lambda}+\left(t_{\sigma \lambda}^{\prime}+t_{\sigma \bar{\lambda}}\right) f_{\lambda}^{\dagger}\right]-i\left[\left(t_{\sigma \lambda}^{\prime *}+t_{\sigma \bar{\lambda}}^{*}\right) f_{\lambda}+\left(t_{\sigma \lambda}^{\prime *}-t_{\sigma \bar{\lambda}}^{*}\right) f_{\lambda}^{\dagger}\right] d_{\sigma} \\
& =\sum_{\sigma, \lambda}-i t_{\sigma \lambda} d_{\sigma}^{\dagger} f_{\lambda}+i t_{\sigma \lambda-}^{*} f_{\lambda}^{\dagger} d_{\sigma}+i t_{\sigma \lambda+} d_{\sigma}^{\dagger} f_{\lambda}^{\dagger}-i t_{\sigma \lambda+}^{*} f_{\lambda} d_{\sigma},
\end{aligned}
$$


where we have defined $t_{\sigma \lambda \pm}=t_{\sigma \bar{\lambda}} \pm t_{\sigma \lambda}^{\prime}$. Furthermore, we redefine the MBS coupling in the wire so that $H_{M}=\sum_{\lambda} \delta_{\lambda}\left(2 f_{\lambda}^{\dagger} f_{\lambda}-1\right)$, where $\delta_{r}\left(\delta_{l}\right)$ now parametrizes the overlap between the inner (outer) MBSs. With this redefinition, we see that the transformed Hamiltonian is, term by term, identical to Eq. (2) with the exchange of tilded to untilded variables. Therefore, upon performing the same Schrieffer-Wolff transformation we find

$$
\begin{aligned}
\mathcal{H}_{s}= & \sum_{\rho \lambda}\left[2 n_{\rho}\left(\frac{\left|t_{\rho \lambda+}\right|^{2}}{\epsilon_{\rho}+2 \delta_{\lambda}}-\frac{\left|t_{\rho \lambda-}\right|^{2}}{\epsilon_{\rho}-2 \delta_{\lambda}}\right)+d_{\rho}^{\dagger} d_{\bar{\rho}}\left(\frac{t_{\bar{\rho} \lambda+}^{*} t_{\rho \lambda+}}{\epsilon_{\bar{\rho}}+2 \delta_{\lambda}}+\frac{t_{\bar{\rho} \lambda+}^{*} t_{\rho \lambda+}}{\epsilon_{\rho}+2 \delta_{\lambda}}-\frac{t_{\bar{\rho} \lambda-}^{*} t_{\rho \lambda-}}{\epsilon_{\bar{\rho}}-2 \delta_{\lambda}}-\frac{t_{\bar{\rho} \lambda-}^{*} t_{\rho \lambda-}}{\epsilon_{\rho}-2 \delta_{\lambda}}\right)\right] f_{\lambda}^{\dagger} f_{\lambda} \\
+ & 2 n_{\rho} \frac{\left|t_{\rho \lambda-}\right|^{2}}{\epsilon_{\rho}-2 \delta_{\lambda}}+d_{\rho}^{\dagger} d_{\bar{\rho}}\left(\frac{t_{\bar{\rho} \lambda-}^{*} t_{\rho \lambda-}}{\epsilon_{\bar{\rho}}-2 \delta_{\lambda}}+\frac{t_{\bar{\rho} \lambda-}^{*} t_{\rho \lambda-}}{\epsilon_{\rho}-2 \delta_{\lambda}}\right), \\
\mathcal{H}_{o}= & \sum_{\rho \lambda}\left\{\left[\left(\frac{1}{\epsilon_{\rho}+2 \delta_{\bar{\lambda}}}+\frac{1}{\epsilon_{\rho}+2 \delta_{\lambda}}\right) t_{\rho \lambda+} t_{\rho \bar{\lambda}+}^{*}-\left(\frac{1}{\epsilon_{\rho}-2 \delta_{\lambda}}+\frac{1}{\epsilon_{\rho}-2 \delta_{\bar{\lambda}}}\right) t_{\rho \bar{\lambda}-} t_{\rho \lambda-}^{*}\right] n_{\rho}\right. \\
& \left.+\left[\left(\frac{1}{\epsilon_{\bar{\rho}}+2 \delta_{\bar{\lambda}}}+\frac{1}{\epsilon_{\rho}+2 \delta_{\lambda}}\right) t_{\bar{\rho} \bar{\lambda}+}^{*} t_{\rho \lambda+}-\left(\frac{1}{\epsilon_{\bar{\rho}}-2 \delta_{\lambda}}+\frac{1}{\epsilon_{\rho}-2 \delta_{\bar{\lambda}}}\right) t_{\bar{\rho} \lambda-}^{*} t_{\rho \bar{\lambda}-}\right] d_{\rho}^{\dagger} d_{\bar{\rho}}\right\} f_{\lambda}^{\dagger} f_{\bar{\lambda}} .
\end{aligned}
$$

In the case considered in the main text, we consider coupling only to the inner MBSs, so that $t_{\sigma l}^{\prime}=t_{\sigma r}=0$, $t_{\sigma l \pm=0}$, and thus $\mathcal{H}_{o}=0$ and

$$
\mathcal{H}_{s}=\sum_{\rho}\left[2 n_{\rho} \frac{\left|t_{\rho+}\right|^{2}}{\epsilon_{\rho}+2 \delta}+d_{\rho}^{\dagger} d_{\bar{\rho}}\left(\frac{t_{\bar{\rho}+}^{*} t_{\rho+}}{\epsilon_{\bar{\rho}}+2 \delta}+\frac{t_{\bar{\rho}+}^{*} t_{\rho+}}{\epsilon_{\rho}+2 \delta}\right)\right] f^{\dagger} f+\left[2 n_{\rho} \frac{\left|t_{\rho-}\right|^{2}}{\epsilon_{\rho}-2 \delta}+d_{\rho}^{\dagger} d_{\bar{\rho}}\left(\frac{t_{\bar{\rho}-}^{*} t_{\rho-}}{\epsilon_{\bar{\rho}}-2 \delta}+\frac{t_{\bar{\rho}-}^{*} t_{\rho-}}{\epsilon_{\rho}-2 \delta}\right)\right] f f^{\dagger},
$$

where $t_{\sigma \pm} \equiv t_{\sigma r \pm}, \delta \equiv \delta_{r}$, and $f \equiv f_{r}$. Performing the summation in spin, we find

$$
\begin{aligned}
& \sum_{\rho} 2 n_{\rho} \frac{\left|t_{\rho \pm}\right|^{2}}{\epsilon_{\rho} \pm 2 \delta}=2 n_{\uparrow} \frac{\left|t_{\uparrow \pm}\right|^{2}}{\epsilon_{\uparrow} \pm 2 \delta} \pm 2 n_{\downarrow} \frac{\left|t_{\downarrow \pm}\right|^{2}}{\epsilon_{\downarrow} \pm 2 \delta}=\left(S_{0}+S_{x}\right) \frac{\left|t_{\uparrow \pm}\right|^{2}}{\epsilon_{\uparrow} \pm 2 \delta}+\left(S_{0}-S_{x}\right) \frac{\left|t_{\downarrow \pm}\right|^{2}}{\epsilon_{\downarrow} \pm 2 \delta} \\
& =S_{0}\left(\frac{\left|t_{\uparrow \pm}\right|^{2}}{\epsilon_{\uparrow} \pm 2 \delta}+\frac{\left|t_{\downarrow \pm}\right|^{2}}{\epsilon_{\downarrow} \pm 2 \delta}\right)+S_{x}\left(\frac{\left|t_{\uparrow \pm}\right|^{2}}{\epsilon_{\uparrow} \pm 2 \delta}-\frac{\left|t_{\downarrow \pm}\right|^{2}}{\epsilon_{\downarrow} \pm 2 \delta}\right) \equiv S_{0} B_{0}^{ \pm}+S_{x} B_{x}^{ \pm}, \\
& \sum_{\rho} d_{\rho}^{\dagger} d_{\bar{\rho}}\left(\frac{t_{\bar{\rho}+}^{*} t_{\rho+}}{\epsilon_{\bar{\rho}} \pm 2 \delta}+\frac{t_{\bar{\rho}+}^{*} t_{\rho+}}{\epsilon_{\rho} \pm 2 \delta}\right)=d_{\uparrow}^{\dagger} d_{\downarrow}\left(\frac{t_{\downarrow \pm}^{*} t_{\uparrow \pm}}{\epsilon_{\downarrow} \pm 2 \delta}+\frac{t_{\downarrow \pm}^{*} t_{\uparrow \pm}}{\epsilon_{\uparrow} \pm 2 \delta}\right)+d_{\downarrow}^{\dagger} d_{\uparrow}\left(\frac{t_{\uparrow \pm}^{*} t_{\downarrow \pm}}{\epsilon_{\uparrow} \pm 2 \delta}+\frac{t_{\uparrow \pm}^{*} t_{\downarrow \pm}}{\epsilon_{\downarrow} \pm 2 \delta}\right) \\
& =\frac{-S_{z}+i S_{y}}{2}\left(\frac{t_{\downarrow \pm}^{*} t_{\uparrow \pm}}{\epsilon_{\downarrow} \pm 2 \delta}+\frac{t_{\downarrow \pm}^{*} t_{\uparrow \pm}}{\epsilon_{\uparrow} \pm 2 \delta}\right)-\frac{S_{z}+i S_{y}}{2}\left(\frac{t_{\uparrow \pm}^{*} t_{\downarrow \pm}}{\epsilon_{\uparrow} \pm 2 \delta}+\frac{t_{\uparrow \pm}^{*} t_{\downarrow \pm}}{\epsilon_{\downarrow} \pm 2 \delta}\right) \\
& =-\frac{S_{z}}{2}\left(\frac{t_{\downarrow \pm}^{*} t_{\uparrow \pm}}{\epsilon_{\downarrow} \pm 2 \delta}+\frac{t_{\downarrow \pm}^{*} t_{\uparrow \pm}}{\epsilon_{\uparrow} \pm 2 \delta}+\frac{t_{\uparrow \pm}^{*} t_{\downarrow \pm}}{\epsilon_{\uparrow} \pm 2 \delta}+\frac{t_{\uparrow \pm}^{*} t_{\downarrow \pm}}{\epsilon_{\downarrow} \pm 2 \delta}\right) \\
& +i \frac{S_{y}}{2}\left(\frac{t_{\downarrow \pm}^{*} t_{\uparrow \pm}}{\epsilon_{\downarrow} \pm 2 \delta}+\frac{t_{\downarrow \pm}^{*} t_{\uparrow \pm}}{\epsilon_{\uparrow} \pm 2 \delta}-\frac{t_{\uparrow \pm}^{*} t_{\downarrow \pm}}{\epsilon_{\uparrow} \pm 2 \delta}-\frac{t_{\uparrow \pm}^{*} t_{\downarrow \pm}}{\epsilon_{\downarrow} \pm 2 \delta}\right) \\
& =-S_{z} \operatorname{Re}\left(t_{\uparrow \pm}^{*} t_{\downarrow \pm}\right)\left(\frac{1}{\epsilon_{\uparrow} \pm 2 \delta}+\frac{1}{\epsilon_{\downarrow} \pm 2 \delta}\right)+S_{y} \operatorname{Im}\left(t_{\uparrow \pm}^{*} t_{\downarrow \pm}\right)\left(\frac{1}{\epsilon_{\uparrow} \pm 2 \delta}+\frac{1}{\epsilon_{\downarrow} \pm 2 \delta}\right) \\
& \equiv S_{z} B_{z}^{ \pm}+S_{y} B_{y}^{ \pm} \text {. }
\end{aligned}
$$

Upon identifying $t_{\sigma r}^{\prime}=t_{\sigma r} / i$ and $\mathcal{H}_{T}=\mathcal{H}_{s}$, we obtain Eq. (16) with effective magnetic field $B_{i}^{ \pm}$given by Eq. (17).

\section{APPENDIX C: NUMERICAL CALCULATION OF SPIN ON THE DOT}

In this Appendix we plot the $x$ component of spin on the dot $S_{j, x}$ as a function of position defined by $S_{j, x}=\tilde{Y}_{j}^{\dagger} \hat{S}_{x} \tilde{Y}_{j}$ (in units of $\hbar / 2$ ), with $\tilde{Y}_{j}$ the dot wave function at site $j$ for the lowest positive energy level of the dot found numerically (see Fig. 11). Here the quantum dot is far away from MBSs, so the only nonzero spin projection of the dot level is $S_{x}$, see Fig. 10. In general, the spin oscillates at a period set by the SOI. For weak magnetic fields, these oscillations are close to be symmetric around zero so that the average spin projection on the dot is almost zero [see Fig. 11 (left panel)]. For strong magnetic fields, there is asymmetry 

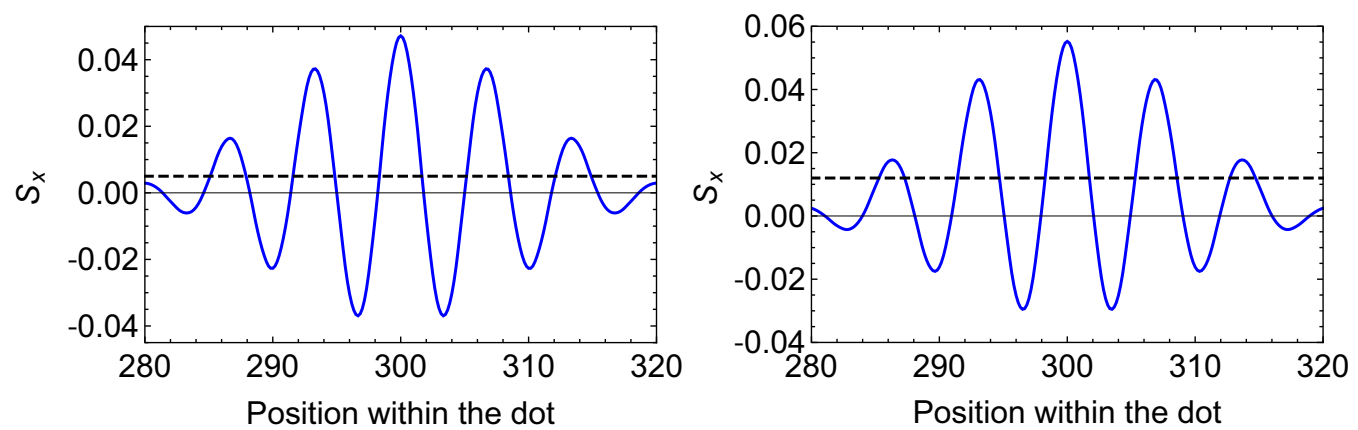

FIG. 11. The spin component $S_{x}$ of the lowest positive energy level of the dot (blue solid line) as a function of the position within the dot when the magnetic field is weak $\left(\Delta_{Z}=0.04\right.$, left panel) and strong $\left(\Delta_{Z}=0.12\right.$, right panel). The black dashed line stands for the symmetric axis of the blue curve corresponding to the average spin projection $S_{x}$ on the dot. The system parameters are the same as in Fig. 9.

around zero, resulting in the average spin polarization along the magnetic field [see Fig. 11 (right panel)]. This explains the offset in $S_{x}$ component of the average spin of the dot shown in Fig. 10 in the main text [44].

[1] V. Mourik, K. Zuo, S. Frolov, S. Plissard, E. Bakkers, and L. Kouwenhoven, Science 336, 1003 (2012).

[2] A. Das, Y. Ronen, Y. Most, Y. Oreg, M. Heiblum, and H. Shtrikman, Nat. Phys. 8, 887 (2012).

[3] L. P. Rokhinson, X. Liu, and J. K. Furdyna, Nat. Phys. 8, 795 (2012).

[4] M. T. Deng, C. L. Yu, G. Y. Huang, M. Larsson, P. Caroff, and H. Q. Xu, Nano Lett. 12, 6414 (2012).

[5] A. D. K. Finck, D. J. Van Harlingen, P. K. Mohseni, K. Jung, and X. Li, Phys. Rev. Lett. 110, 126406 (2013).

[6] H. O. H. Churchill, V. Fatemi, K. Grove-Rasmussen, M. T. Deng, P. Caroff, H. Q. Xu, and C. M. Marcus, Phys. Rev. B 87, 241401 (2013).

[7] S. Nadj-Perge, I. K. Drozdov, J. Li, H. Chen, S. Jeon, J. Seo, A. H. MacDonald, B. A. Bernevig, and A. Yazdani, Science 346, 602 (2014).

[8] R. Pawlak, M. Kisiel, J. Klinovaja, T. Meier, S. Kawai, T. Glatzel, D. Loss, and E. Meyer, Npj Quant. Info. 2, 16035(E) (2016).

[9] A. Y. Kitaev, Ann. Phys. 303, 2 (2003).

[10] H. M. Freedman, A. Kitaev, and Z. Wang, Commun. Math. Phys. 227, 587 (2002).

[11] S. B. Bravyi and A. Y. Kitaev, Ann. Phys. 298, 210 (2002).

[12] M. H. Freedman, A. Kitaev, M. J. Larsen, and Z. Wang, Bull. Am. Math. Soc. (N.S.) 40, 31 (2003).

[13] A. Y. Kitaev, Phys. Usp. 44, 131 (2001).

[14] S. Bravyi, Phys. Rev. A 73, 042313 (2006).

[15] T. Hyart, B. van Heck, I. C. Fulga, M. Burrello, A. R. Akhmerov, and C. W. J. Beenakker, Phys. Rev. B 88, 035121 (2013).

[16] M. Leijnse and K. Flensberg, Phys. Rev. Lett. 107, 210502 (2011).

[17] M. Leijnse and K. Flensberg, Phys. Rev. B 86, 104511 (2012).

[18] S. Plugge, L. A. Landau, E. Sela, A. Altland, K. Flensberg, and R. Egger, Phys. Rev. B 94, 174514 (2016).

[19] S. Hoffman, C. Schrade, J. Klinovaja, and D. Loss, Phys. Rev. B 94, 045316 (2016).

[20] L. A. Landau, S. Plugge, E. Sela, A. Altland, S. M. Albrecht, and R. Egger, Phys. Rev. Lett. 116, 050501 (2016).
[21] T. Karzig, C. Knapp, R. M. Lutchyn, P. Bonderson, M. Hastings, C. Nayak, J. Alicea, K. Flensberg, S. Plugge, Y. Oreg, C. M. Marcus, and M. H. Freedman, Phys. Rev. B 95, 235305 (2017).

[22] S. Plugge, A. Rasmussen, R. Egger, and K. Flensberg, New J. Phys. 19, 012001 (2017).

[23] R. M. Lutchyn, J. D. Sau, and S. D. Sarma, Phys. Rev. Lett. 105, 077001 (2010).

[24] Y. Oreg, G. Refael, and F. von Oppen, Phys. Rev. Lett. 105, 177002 (2010).

[25] S. M. Albrecht, A. P. Higginbotham, M. Madsen, F. Kuemmeth, T. S. Jespersen, J. Nygård, P. Krogstrup, and C. M. Marcus, Nature (London) 531, 206 (2016).

[26] D. Loss and D. P. DiVincenzo, Phys. Rev. A 57, 120 (1998).

[27] C. Kloeffel and D. Loss, Annu. Rev. Condens. Matter Phys. 4, 51 (2013).

[28] K. Flensberg, Phys. Rev. Lett. 106, 090503 (2011).

[29] D. E. Liu and H. U. Baranger, Phys. Rev. B 84, 201308(R) (2011).

[30] E. Vernek, P. H. Penteado, A. C. Seridonio, and J. C. Egues, Phys. Rev. B 89, 165314 (2014).

[31] M. Deng, S. Vaitiekènas, E. B. Hansen, J. Danon, M. Leijnse, K. Flensberg, J. Nygård, P. Krogstrup, and C. M. Marcus, Science 354, 1557 (2016).

[32] L. S. Ricco, Y. Marques, F. A. Dessotti, R. S. Machado, M. de Souza, and A. C. Seridonio, Phys. Rev. B 93, 165116 (2016).

[33] F. A. Dessotti, L. S. Ricco, Y. Marques, L. H. Guessi, M. Yoshida, M. S. Figueira, M. de Souza, P. Sodano, and A. C. Seridonio, Phys. Rev. B 94, 125426 (2016).

[34] C. Schrade, S. Hoffman, and D. Loss, Phys. Rev. B 95, 195421 (2017).

[35] L. Xu, X.-Q. Li, and Q.-F. Sun, J. Phys. Condens. Matter 29, 195301 (2017).

[36] L. H. Guessi, F. A. Dessotti, Y. Marques, L. S. Ricco, G. M. Pereira, P. Menegasso, M. de Souza, and A. C. Seridonio, Phys. Rev. B 96, 041114(R) (2017).

[37] E. Prada, R. Aguado, and P. San-Jose, arXiv:1702.02525.

[38] M. Sato and S. Fujimoto, Phys. Rev. B 79, 094504 (2009).

[39] J. Klinovaja and D. Loss, Phys. Rev. B 86, 085408 (2012). 
[40] J. Kammhuber, M. C. Cassidy, F. Pei, M. P. Nowak, A. Vuik, D. Car, S. R. Plissard, E. P. A. M. Bakkers, M. Wimmer, and L. P. Kouwenhoven, arXiv:1701.06878.

[41] B. Braunecker, G. I. Japaridze, J. Klinovaja, and D. Loss, Phys. Rev. B 82, 045127 (2010).

[42] M. Ruby, F. Pientka, Y. Peng, F. von Oppen, B. W. Heinrich, and K. J. Franke, Phys. Rev. Lett. 115, 197204 (2015).

[43] D. Chevallier and J. Klinovaja, Phys. Rev. B 94, 035417 (2016).

[44] M. Trif, V. N. Golovach, and D. Loss, Phys. Rev. B 77, 045434 (2008).

[45] E. Prada, P. San-Jose, and R. Aguado, Phys. Rev. B 86, 180503 (2012).

[46] D. Rainis, L. Trifunovic, J. Klinovaja, and D. Loss, Phys. Rev. B 87, 024515 (2013).
[47] A. A. Zyuzin, D. Rainis, J. Klinovaja, and D. Loss, Phys. Rev. Lett. 111, 056802 (2013).

[48] J. Schrieffer and P. Wolff, Phys. Rev. 149, 491 (1966).

[49] S. Nadj-Perge, I. K. Drozdov, B. A. Bernevig, and A. Yazdani, Phys. Rev. B 88, 020407 (2013).

[50] J. Klinovaja, P. Stano, A. Yazdani, and D. Loss, Phys. Rev. Lett. 111, 186805 (2013).

[51] B. Braunecker and P. Simon, Phys. Rev. Lett. 111, 147202 (2013).

[52] M. M. Vazifeh and M. Franz, Phys. Rev. Lett. 111, 206802 (2013).

[53] J. Klinovaja and D. Loss, Eur. Phys. J. B 88, 62 (2015).

[54] S. Bravyi, D. P. DiVincenzo, and D. Loss, Ann. Phys. 326, 2793 (2011). 